\title{
Cavity Casimir-Polder Forces and Their Effects in Ground-State Chemical Reactivity
}

\author{
Javier Galego, ${ }^{1}$ Clàudia Climent, ${ }^{1}$ Francisco J. Garcia-Vidal, ${ }^{1,2}$ and Johannes Feist ${ }^{1, *}$ \\ ${ }^{1}$ Departamento de Física Teórica de la Materia Condensada and Condensed Matter Physics Center \\ (IFIMAC), Universidad Autónoma de Madrid, E-28049 Madrid, Spain \\ ${ }^{2}$ Donostia International Physics Center (DIPC), E-20018 Donostia/San Sebastian, Spain
}

(Received 27 July 2018; revised manuscript received 11 February 2019; published 21 June 2019)

\begin{abstract}
Here, we present a fundamental study on how the ground-state chemical reactivity of a single molecule can be modified in a QED scenario, i.e., when it is placed inside a nanoscale cavity and there is strong coupling between the cavity field and vibrational modes within the molecule. We work with a model system for the molecule (Shin-Metiu model) in which nuclear, electronic, and photonic degrees of freedom are treated on the same footing. This simplified model allows the comparison of exact quantum reaction rate calculations with predictions emerging from transition state theory based on the cavity BornOppenheimer approach. We demonstrate that QED effects are indeed able to significantly modify activation barriers in chemical reactions and, as a consequence, reaction rates. The critical physical parameter controlling this effect is the permanent dipole of the molecule and how this magnitude changes along the reaction coordinate. We show that the effective coupling can lead to significant single-molecule energy shifts in an experimentally available nanoparticle-on-mirror cavity. We then apply the validated theory to a realistic case (internal rotation in the 1,2-dichloroethane molecule), showing how reactions can be inhibited or catalyzed depending on the profile of the molecular dipole. Furthermore, we discuss the absence of resonance effects in the present scenario, which can be understood through its connection to Casimir-Polder forces. Finally, we treat the case of many-molecule strong coupling and find collective modifications of reaction rates if the molecular permanent dipole moments are oriented with respect to the cavity field.
\end{abstract}

DOI: 10.1103/PhysRevX.9.021057

Subject Areas: Atomic and Molecular Physics, Chemical Physics, Photonics

\section{INTRODUCTION}

The field of (nonrelativistic) cavity quantum electrodynamics (CQED) has proved that the quantum nature of light can be exploited for many interesting applications that involve the modifications of material properties in one way or another $[1,2]$. In this context, strong light-matter coupling is particularly appealing [3]. The regime of strong coupling is achieved when the coherent energy exchange between the excitations of a material (excitons) and of the cavity light modes is faster than the decay rate of either constituent. The resulting excitations are the well-known polaritons, which combine properties of both light and matter, leading to many interesting applications (see Ref. [4] for a recent review). In recent years, strong coupling to organic materials has received great attention

*johannes.feist@uam.es

Published by the American Physical Society under the terms of the Creative Commons Attribution 4.0 International license. Further distribution of this work must maintain attribution to the author(s) and the published article's title, journal citation, and DOI. for its potential to greatly influence fundamental features of the underlying organic molecules such as their optical response [5-7], transport properties [8-12], or chemical reactivity [13-15]. In particular, the potential of polaritonic chemistry, i.e., the ability to influence the chemical structure and reactions of organic compounds through coupling to a cavity, has attracted a lot of interest [16-34].

Most of the research on polaritonic chemistry with organic molecules deals with electronic strong coupling. This phenomenon leads to many interesting effects such as collective protection of polaritons and changes in chemical reactivity $[19,20]$, cavity-induced nonadiabatic phenomena $[21,28,34]$, and the opening of novel reaction pathways in photochemistry [23].

More recently, the possibility of influencing the thermally driven reactivity of organic molecules in the ground state has been demonstrated by coupling the cavity to vibrational transitions of the molecules [14,35-37]. This opens a wide range of possibilities due to the fact that no external input of energy is needed at all, such as cavity-enabled catalysis and manipulation of ground-state chemical reactions. Cavityinduced modifications to the ground state have also been theoretically studied. In particular, for model molecules 
without ground-state dipole moments and only electronic dipole transitions, it has been shown that there is no collective enhancement of energy shifts [17] and, more specifically, that chemical reactions are not strongly modified even under ultrastrong collective coupling [30]. In a series of papers based on more microscopic models, Flick and co-workers showed that ground-state properties can be significantly modified under single-molecule (ultra)strong coupling $[18,25,26]$ but did not treat chemical reactivity.

In the present work, we aim to understand cavityinduced modifications of ground-state chemistry in coupled molecule-cavity systems. This paper is structured as follows: In Sec. II, we present the light-matter interaction Hamiltonian for a single molecule coupled to a nanoscale cavity. After a brief discussion of the validity of this Hamiltonian, we study a simple model system, the ShinMetiu model, and in Sec. III obtain the cavity-modified reactivity from formally exact quantum rate calculations [38-40]. In Sec. IV, we develop a simplified theory that allows us to understand ground-state chemical reactivity changes based on well-known concepts such as transition state theory (TST) [41,42] by exploiting the cavity BornOppenheimer approximation [25]. We show in Sec. V that, to a good approximation, perturbation theory can be used to predict cavity-induced chemical changes in terms of baremolecule ground-state properties and also allows us to make explicit connections to electrostatic, van der Waals, and Casimir-Polder interactions. This theory is exploited in Sec. VI to demonstrate that, for a realistic experimental geometry, a multimode nanoparticle-on-mirror cavity [43-45], the effective single-molecule coupling can be significant. In Sec. VII, we study the modification of reaction rates in the 1,2-dichloroethane molecule, demonstrating the potential of a cavity to catalyze or inhibit reactions or even to modify the equilibrium configuration of the molecule. In Sec. VIII, we discuss in detail the dependence of chemical reaction rates on the frequency of the cavity mode. We observe that, in contrast to polariton formation, which requires the cavity photon and molecular excitations to be resonant, no such requirement exists for the change of reaction rates in the cavity. In the case of a single molecule as treated up to that point, the coupling strengths required to obtain significant changes in chemical reactivity correspond to the most tightly confined plasmonic nanogap cavities available experimentally [43-46]. In Sec. IX, we thus extend our model to an ensemble of molecules and find a collective enhancement of the effect under orientational alignment of the molecular dipoles.

We mention here that we do not explicitly treat the case of many molecules coupled to a cavity with a continuum of modes, i.e., the case which corresponds to the experimentally used Fabry-Perot cavities with in-plane dispersion [14,37]. We also do not explicitly treat solvent effects. While these are well known to be important in chemical reactions, their effect depends strongly on the chosen solvent and experimental setup (particularly in nanocavities). However, we mention that the latest experimental studies indicate that solvent effects might be responsible and/or relevant for the experimentally observed resonance-dependent ground-state chemical reactivity $[35,36]$.

\section{THEORY AND MODEL SYSTEM}

\section{A. Light-matter Hamiltonian}

We start from the general nonrelativistic light-matter Hamiltonian of QED in minimal coupling, describing a collection of charged particles coupled to the electromagnetic (EM) field. Here and in the following, we use atomic units $\left(\hbar=4 \pi \epsilon_{0}=m_{e}=1\right)$ unless stated otherwise.

$$
\begin{aligned}
\hat{H}= & \sum_{i} \frac{\left[\hat{\mathbf{p}}_{i}-Q_{i} \hat{\mathbf{A}}\left(\mathbf{r}_{i}\right)\right]^{2}}{2 m_{i}}+\sum_{i>j} \frac{Q_{i} Q_{j}}{\left|\mathbf{r}_{i}-\mathbf{r}_{j}\right|} \\
& +\frac{1}{8 \pi} \int\left[\hat{\mathbf{E}}^{\perp}(\mathbf{r})^{2}+c^{2} \hat{\mathbf{B}}(\mathbf{r})^{2}\right] d^{3} \mathbf{r},
\end{aligned}
$$

where $\hat{\mathbf{E}}^{\perp}(\mathbf{r})=-(1 / c)[\partial \hat{\mathbf{A}} / \partial t]$ is the transverse part of the electric field (with the longitudinal part responsible for the instantaneous Coulomb interaction $Q_{i} Q_{j} / r_{i j}$ ) and we use the Coulomb gauge $\nabla \cdot \hat{\mathbf{A}}=0$. We note explicitly that, here, the EM operators represent free-space modes (i.e., without boundary conditions imposing a cavity structure), while the collection of charged particles (specifically, electrons and nuclei) represents both the material part of the cavity (e.g., mirrors) and the emitters (such as molecules). In particular, the cavity material together with the EM-field modes will have approximately bosonic eigenmodes that can be identified as the "cavity modes" and, in general, will be given by superpositions of material and EM-field excitations [47], as explicitly shown for plasmonic systems in Ref. [48]. For simplicity and generality, in the following, we assume that the cavity-molecule system we are treating is well described within the quasistatic approximation, which applies when all distances in the problem are significantly smaller than the relevant wavelengths. In particular, this is a good approximation for small plasmon- and phonon-polariton nanoantennas and nanoresonators, which are the only currently available systems that achieve a strong enough field concentration to obtain strong single-emitter couplings with "real" atoms or molecules [43,49-52] (as opposed to "artificial atoms" such as superconducting qubits [53-55]). In the quasistatic limit, the transversal fields are negligible, [56] so that $\hat{\mathbf{A}}=\hat{\mathbf{B}}=\hat{\mathbf{E}}^{\perp} \approx 0$, and the Hamiltonian simply becomes

$$
\hat{H}=\sum_{i} \frac{\hat{\mathbf{p}}_{i}^{2}}{2 m_{i}}+\sum_{i>j} \frac{Q_{i} Q_{j}}{\left|\mathbf{r}_{i}-\mathbf{r}_{j}\right|},
$$


with the sums over $i$ and $j$ still including all particles in the (nano)cavity as well as the molecules. We next separate the particles into several groups: one containing the cavity material and one for each molecule. We assume that the cavity material is "macroscopic" enough that it responds linearly to external fields $[47,48,57-60]$ and can, thus, be well described by a collection of bosonic modes with frequencies $\omega_{k}$ and annihilation operators $a_{k}$ (e.g., corresponding to the "instantaneous" plasmon modes in Ref. [48]). For simplicity, we first consider a single molecule including $n_{e}$ electrons and $n_{n}$ nuclei. The Hamiltonian then becomes

$$
\begin{aligned}
\hat{H}= & \sum_{i=1}^{n_{n}} \frac{\hat{\mathbf{P}}_{i}^{2}}{2 M_{i}}+\hat{H}_{e}(\hat{\mathbf{x}}, \hat{\mathbf{R}})+\sum_{k} \omega_{k} \hat{a}_{k}^{\dagger} \hat{a}_{k} \\
& +\sum_{k}\left(\hat{a}_{k}+\hat{a}_{k}^{\dagger}\right) \sum_{j} Q_{j} \phi_{k}\left(\hat{\mathbf{r}}_{j}\right) .
\end{aligned}
$$

The bare molecular Hamiltonian corresponds to the first two terms: the kinetic energy of $n_{n}$ nuclei and the electronic Hamiltonian. The latter includes the kinetic energy of the $n_{e}$ electrons and the nucleus-nucleus, electron-electron, and nucleus-electron interaction potentials. This operator depends on all the electronic and nuclear positions, $\hat{\mathbf{x}}=$ $\left(\hat{\mathbf{x}}_{1}, \hat{\mathbf{x}}_{2}, \ldots, \hat{\mathbf{x}}_{n_{e}}\right)$ and $\hat{\mathbf{R}}=\left(\hat{\mathbf{R}}_{1}, \hat{\mathbf{R}}_{2}, \ldots, \hat{\mathbf{R}}_{n_{n}}\right)$, respectively. The following two terms correspond to the bosonic cavity modes and the interaction of the molecular charges (with $j$ running over both electrons and nuclei) with the electrostatic potential $\phi_{k}(\mathbf{r})$, i.e., the Coulomb potential corresponding to the charge distribution of each cavity mode. By performing a multipole expansion of the molecular charges and assuming that the molecule is uncharged and sufficiently localized, this term can be well approximated by $\hat{\boldsymbol{\mu}} \cdot \hat{\mathbf{E}}\left(\mathbf{r}_{m}\right)$, i.e., the interaction of the molecular dipole with the cavity electric field (the gradient of the potential) at the position $\mathbf{r}_{m}$ of the molecule, which we write as

$$
\left(\hat{a}_{k}+\hat{a}_{k}^{\dagger}\right) \sum_{j} Q_{j} \phi_{k}\left(\hat{\mathbf{r}}_{j}\right) \approx \omega_{k} \hat{q}_{k} \boldsymbol{\lambda}_{k} \cdot \hat{\boldsymbol{\mu}}(\hat{\mathbf{x}}, \hat{\mathbf{R}}),
$$

where $\hat{q}_{k}=\left(1 / \sqrt{2 \omega_{k}}\right)\left(\hat{a}_{k}+\hat{a}_{k}^{\dagger}\right)$ is the position operator of the harmonic oscillator and the electric field strength is determined by $\lambda_{k}=\lambda_{k} \mathbf{\epsilon}_{k}$, with polarization vector $\mathbf{\epsilon}$. The coupling constant can be related to both the singlephoton electric field strength and the (position-dependent) effective mode volume of the quantized mode, with $\lambda_{k}=$ $\sqrt{2 / \omega_{k}} E_{1 \mathrm{ph}, k}\left(\mathbf{r}_{m}\right)=\sqrt{4 \pi / V_{\text {eff }, k}}$. Here, the effective EM mode volume is defined as $V_{\text {eff }, k}=\int \varepsilon(\mathbf{r})^{2}|\mathbf{E}(\mathbf{r})|^{2} d^{3} \mathbf{r} /$ $\left[\varepsilon\left(\mathbf{r}_{m}\right)^{2}\left|\mathbf{E}\left(\mathbf{r}_{m}\right)\right|^{2}\right]$, although the normalization integral formally diverges for lossy modes and has to be properly generalized [48,61-63].

The proper description of the light-matter interaction Hamiltonian under (ultra)strong-coupling conditions is a very active topic of discussion in the literature [64-72].
In particular, much of this discussion centers on the importance of the so-called dipole self-energy term $\frac{1}{2}[\boldsymbol{\lambda} \cdot \hat{\boldsymbol{\mu}}(\hat{\mathbf{x}}, \hat{\mathbf{R}})]^{2}$ that arises in the Power-Zienau-Woolley transformation, where the interaction with the transversal field $\hat{\mathbf{A}}$ is transformed to an electric-field-dipole interaction with the same form as Eq. (4) plus the above-mentioned dipole self-energy term. As we have discussed, and as is well known in the literature on macroscopic QED [73], this term does not appear for interactions with purely longitudinal modes that are well described within the quasistatic approximation, i.e., in situations where retardation and propagation effects of the EM fields can be neglected. Given the fact that reaching strong or ultrastrong coupling with one (or a few) atoms or molecules requires strongly subwavelength mode volumes, $V_{\text {eff }, k} \ll\left(2 \pi c / \omega_{k}\right)^{3}$, it follows that the quasistatic approximation should be applicable for most realistic cavities with few-emitter strong coupling. On the other hand, this extreme field localization also can require going beyond the point-dipole interaction either by directly using the interaction with the full spacedependent potential $\phi_{k}(\mathbf{r})$ [74] or by including higher multipoles in Eq. (4) [75]. Doing so also resolves the formal lack of a ground state when the computational box is made too large and no dipole self-energy term is present $[67,76]$.

However, it should be noted that if the sum over cavity modes is truncated and the effect of all but one (or a few) modes is approximately represented by renormalizing the emitter potential (and emitter-emitter interactions in the multiple-emitter case), it is necessary to add back an effective (collective) dipole self-interaction to avoid double counting of modes, as explained in Ref. [66].

We note that, while we explicitly treat a (nano)cavity within the quasistatic approximation, in which the cavity fields can be understood as due to the instantaneous Coulomb interaction between charged particles, it still makes sense to speak of the cavity modes as electromagnetic or photonic modes with an associated electric field. The modes, which physically correspond to, e.g., plasmonic or phonon-polaritonic resonances, can be seen as strongly confined photons. These modes are most easily obtained by solving Maxwell's equations for a given geometry, either numerically or with approaches such as transformation optics [77]. Only in the limit of extremely small nanocavities does it become possible, and sometimes necessary, to treat them explicitly as a collection of nuclei and electrons using ab initio techniques [78-80].

In the following, we first treat a cavity in which only a single mode has significant coupling to the molecule (in Appendix A, we discuss some systems in which this is a valid approximation). Since the interaction depends on the inner product between the electric field and the total dipole moment $\hat{\boldsymbol{\mu}}=\sum_{i}^{n_{n}} Z_{i} \hat{\mathbf{R}}_{i}-\sum_{i}^{n_{e}} \hat{\mathbf{x}}_{i}$, only the projection $\hat{\mu}_{\epsilon}=\hat{\boldsymbol{\epsilon}} \cdot \hat{\boldsymbol{\mu}}$ is relevant, and we have to deal with only scalar quantities. For the sake of simplicity, we rewrite 
$\hat{\mu}_{\epsilon} \rightarrow \hat{\mu}$. We also assume perfect alignment between the molecule and the field unless indicated otherwise.

\section{B. Molecular model}

In order to study changes in ground-state chemical reactivity induced by (vibrational) strong coupling, we first treat a simple molecular model system that is numerically fully solvable and has been extensively studied in model calculations of chemical reaction rates, the ShinMetiu model [81]. It treats three nuclei and one electron moving in one dimension, as presented in Fig. 1(a). Two of the nuclei are separated by a distance $L$ and fixed in place, while the remaining nucleus and the electron are free to move. The repulsive interaction of the mobile nucleus with the fixed ones is given by a normal Coulomb potential, while the attractive electron-nuclei interaction is given by softened Coulomb potentials $V_{\text {en }}\left(r_{i}\right)=\operatorname{Zerf}\left(r_{i} / R_{c}\right) / r_{i}$, where $r_{i}$ is the distance between the electron and nucleus

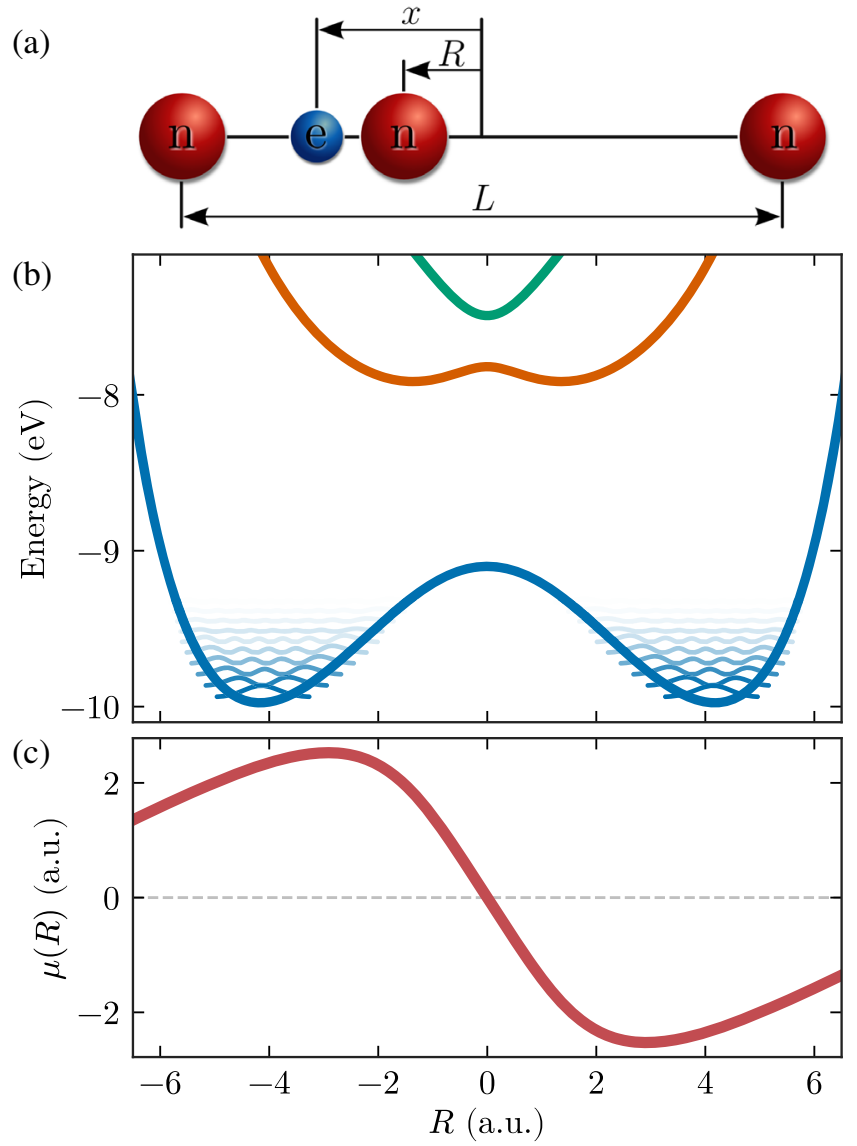

FIG. 1. (a) Schematic representation of the Shin-Metiu model close to one of the equilibrium configurations. The two ions on both sides are fixed at a distance $L$, while the electron and the remaining ion can move freely in between. (b) Potential energy surfaces of the model with the vibrational levels and associated probability densities of the ground state (blue curve) represented. (c) Ground-state dipole moment. $i$ and $R_{c}$ is the softening parameter. The system has two stable nuclear configurations (minima of the ground-state Born-Oppenheimer surface) that represent two different isomers of a charge or proton transfer reaction. Given that the electronic excitation energies and thus the nonadiabatic couplings between different potential energy surfaces can be varied easily by changing the parameters of the Shin-Metiu model, it has been extensively studied in the context of correlated electron-nuclear dynamics [82,83], as well as in the context of polariton formation under strong coupling $[25,26]$. The parameters chosen throughout the present work are $Z=1, L=10 \AA \approx 18.9$ a.u., $M=1836$ a.u., and $R_{c}=1.5 \AA \approx 2.83$ a.u. (for all three nuclei), resulting in the Born-Oppenheimer potential energy surfaces shown in Fig. 1(b), with negligible nonadiabatic coupling between electronic surfaces. The figure also shows the first few vibrational eigenstates close to each minimum (tunneling through the central energy barrier is negligible for these states, so that they can be chosen to be localized on the left or right, respectively). In Fig. 1(c), we show the ground-state permanent dipole moment $\mu_{g}(R)=\langle g|\mu(R)| g\rangle$. Below, we demonstrate that, to a good approximation, the ground-state potential energy surface and dipole moment are sufficient to describe the change in the molecular ground-state structure and chemical reactivity due to the cavity. Additionally, we note here that the light-matter coupling strength for the formation of vibropolaritons, i.e., hybridization of the photon mode with the vibrational transitions of the molecule, is determined by the transition dipole moment and frequency of the quantized vibrational levels of the molecule. Within a lowest-order expansion around the equilibrium position, $V_{g}(R) \approx V_{g}\left(R_{0}\right)+$ $\frac{1}{2} M \omega_{\nu}^{2}\left(R-R_{0}\right)^{2}, \mu_{g}(R) \approx \mu_{g}\left(R_{0}\right)+\mu_{g}^{\prime}\left(R_{0}\right)\left(R-R_{0}\right)$, these are given by $\omega_{\nu}=72.6 \mathrm{meV}$ and $\mu_{\nu} \approx \mu_{g}^{\prime}\left(R_{0}\right) / \sqrt{2 M \omega_{\nu}}$, giving a Rabi frequency $\Omega_{R}=\lambda \mu_{g}^{\prime}\left(R_{0}\right) / \sqrt{M}$ on resonance $\left(\omega_{c}=\omega_{\nu}\right)$ [84].

\section{QUANTUM REACTION RATES}

In this section, we analyze the cavity-induced change in the rate of the ground-state proton-transfer reaction from the left minimum at $R \approx-4$ a.u. to the right one. In the present section, we take advantage of the simplicity of the Shin-Metiu model to exactly compute the quantum reaction rate without any approximations, which automatically takes into account all quantum effects such as tunneling or zero-point energy. We follow the approach of Miller [40], based on the correlation function formalism introduced in Refs. [38,39]. Within this approach, the rate for a molecular reaction is given by

$$
k(T)=\frac{1}{Q_{r}(T)} \int_{0}^{t_{f} \rightarrow \infty} C_{\mathrm{ff}}(t) d t
$$


where $Q_{r}(T)=\operatorname{tr}[\exp (-\beta \hat{H})]$, with $\beta^{-1}=k_{B} T$, is the partition function of the reactants at temperature $T$ and $C_{\mathrm{ff}}(t)$ is the flux-flux autocorrelation function, defined as

$$
C_{\mathrm{ff}}(t)=\operatorname{tr}\left[\bar{F} \hat{U}^{\dagger}\left(t_{c}\right) \bar{F} \hat{U}\left(t_{c}\right)\right]
$$

This correlation function is computed as the trace of a product of operators, where $U\left(t_{c}\right)=\exp \left(-i \hat{H} t_{c}\right)$, with $t_{c}=t-i \beta / 2$, is the complex time evolution operator and $\bar{F}$ represents the symmetrized flux operator

$$
\bar{F}=\frac{1}{2 M}[\hat{P} \delta(s)+\delta(s) \hat{P}]
$$

Here, $\hat{P}$ is the nuclear momentum operator, and the surface dividing the reactant and product states is defined by the zeros of the function $s=s(R)$. In our case, the line that defines products and reactants is $R=0$, i.e., $s(R)=R$. The flux-flux autocorrelation function describes the temporal flux of positive-momenta probability through the dividing surface of a thermally averaged initial state [which is accounted for by the thermal part of the $\hat{U}\left(t_{c}\right)$ operator]. Negative values of $C_{\mathrm{ff}}(t)$ indicate a recrossing of the dividing surface in the opposite direction, thus contributing to a rate decrease. We note that the use of a given temperature $T$ implies that the whole system (molecules and cavity mode) is in thermal equilibrium, as would be achieved by including explicit system-bath interactions (e.g., cavity dissipation), with all baths at the same temperature, and letting the system evolve to the steady state.

In order to obtain the rates of the coupled electronicnuclear-photonic system, we discretize all three degrees of freedom (d.o.f.), using a finite-element discrete variable representation [85] for $x$ and $R$ as well as the Fock basis for the cavity photon mode. This allows us to diagonalize the full Hamiltonian [Eq. (3)] and, thus, to trivially calculate Eq. (6) for arbitrary time $t$. For numerical efficiency, we perform the diagonalization in steps, first diagonalizing the bare molecular Hamiltonian, performing a cutoff in energy, and then diagonalizing the coupled system in this basis. We carefully check convergence with respect to all involved grid and basis set parameters and cutoffs. As is well known [81], due to the absence of dissipation in the model, for large times the correlation function becomes negative and oscillates around zero, corresponding to the wave packet that has crossed the barrier returning back through the dividing surface after reflection at the other side of the potential (at $R \approx 6$ a.u.). However, in a real system, the reaction coordinate is coupled to other vibrational and solvent d.o.f. that will dissipate the energy and prevent recrossing. To represent this effect, we choose a final time $t_{f}$ around which the correlation function stays equal to zero for a while and only integrate up to that time in Eq. (5). The time chosen, $t_{f}=35 \mathrm{fs}$, corresponds to typical dissipation times in condensed phase reactions and is similar to values chosen in the cavity-free case [81].

We now study the cavity-modified chemical reaction rates of the hybrid system for different coupling strengths $\lambda$. We note that a coupling strength of $\lambda=0.035$ a.u. corresponds to a Rabi splitting of $\Omega_{R} \approx 0.10 \omega_{\nu}$ for the first vibrational transition. For the sake of comparison, we mention that single-molecule electronic strong coupling has been achieved with mode volumes of approximately $40 \mathrm{~nm}^{3}$ [43], corresponding to $\lambda \approx 0.007$ a.u., and there are indications that effective subnanometer ${ }^{3}$ mode volumes could be reached due to single-atom hot spots [44,45], which would allow the coupling strength to reach values up to $\lambda \approx 0.05$ a.u. Figure 2 shows the rates in an Arrhenius plot, i.e., the logarithm of the rate divided by the temperature as a function of the inverse temperature. The straight lines in Fig. 2 confirm that the hybrid light-matter system follows the behavior described by the Eyring equation [41], which connects the rate of a chemical reaction with the energy barrier $E_{b}$ that separates reactants from products:

$$
k=\kappa 2 \pi k_{B} T e^{-\left(E_{b} / k_{B} T\right)} .
$$

Here, $\kappa$ is a transmission coefficient, typically considered equal to one if nonadiabatic effects can be neglected close to the transition state. This equation follows from classical transition state theory $[41,42]$ and is often used in the context of chemical kinetics.

We thus observe that, even under vibrational strong coupling and the accompanying formation of vibropolaritons, i.e., hybrid light-matter excitations, the reaction rate can still be described by an effective potential energy barrier. However, the effective height of the energy barrier is modified through the CQED effect of strong coupling, leading (for the studied model) to significantly reduced

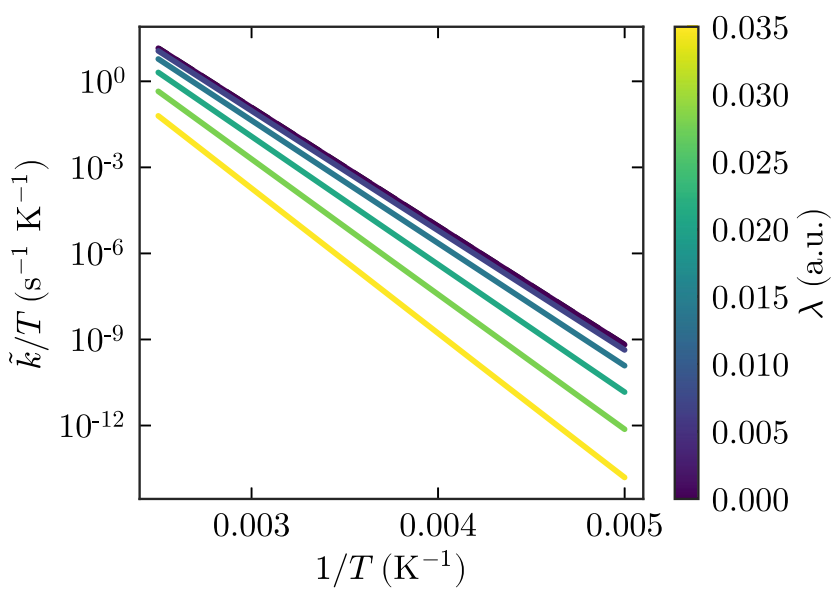

FIG. 2. Arrhenius plot for the rate dependence with the temperature in the hybrid system for several light-matter coupling values. See the main text for details. 
reaction rates. In order to gain further insight into this effect and enable calculations beyond simple model systems, it is desirable to have a theory that is not based on full quantum rate calculations (which require the calculation of nuclear dynamics in $3 N-6$ dimensions). In the next section, we show that this can be achieved by applying (classical) transition state theory to the combined photonic-nuclear potential energy surfaces provided by the cavity BornOppenheimer approximation [26].

\section{CAVITY BORN-OPPENHEIMER APPROXIMATION}

The starting point of the cavity Born-Oppenheimer approximation (CBOA) $[25,26]$ is to write the cavity mode energy as an explicit harmonic oscillator:

$$
\omega_{c}\left(\hat{a}^{\dagger} \hat{a}+\frac{1}{2}\right)=\frac{\hat{p}^{2}}{2}+\omega_{c}^{2} \frac{\hat{q}^{2}}{2},
$$

with $\hat{p}=i \sqrt{\omega_{c} / 2}\left(\hat{a}^{\dagger}-\hat{a}\right)$ and $\hat{q}=\left(1 / \sqrt{2 \omega_{c}}\right)\left(\hat{a}^{\dagger}+\hat{a}\right)$ as discussed in Sec. II. The cavity photon d.o.f. is then treated as nuclearlike and its "kinetic energy" $\hat{p}^{2} / 2$ grouped with the nuclear kinetic energy operators $\sum_{i} \hat{\mathbf{P}}_{i}^{2} /\left(2 M_{i}\right)$ before performing the standard Born-Oppenheimer approximation. This leads to a set of electronic CBO potential energy surfaces (PESs) $\tilde{V}_{i}(\mathbf{R}, q)$ parametric in both nuclear $\mathbf{R}$ and photonic coordinates $q$, obtained by diagonalizing the new electronic Hamiltonian $\hat{H}_{e}(\hat{\mathbf{x}} ; \mathbf{R}, q)=\hat{H}-\left(\hat{p}^{2} / 2\right)-$ $\sum_{i=1}^{n_{n}} \hat{\mathbf{P}}_{i}^{2} /\left(2 M_{i}\right)$ :

$$
\hat{H}_{e}(\hat{\mathbf{x}} ; \mathbf{R}, q) \phi_{i}(\mathbf{x} ; \mathbf{R}, q)=\tilde{V}_{i}(\mathbf{R}, q) \phi_{i}(\mathbf{x} ; \mathbf{R}, q) .
$$

Conceptually, the inclusion of the cavity mode thus simply corresponds to a single additional nuclearlike d.o.f.

The CBOA now consists in neglecting nonadiabatic couplings between different PESs (i.e., neglecting the action of nuclear and photonic kinetic operators on the electronic states) and assuming photonic and nuclear "motion" to proceed on each PES independently. Because of the formal equivalence between nuclear and photonic d.o.f. within this picture, all the standard results of BO theory apply. In particular, the CBOA is a good approximation when the separation between the PESs is larger than typical kinetic energies of the nuclei and the photonic mode. The case of vibrational strong coupling, where the photon energy is comparable to vibrational excitation energies, exactly fulfills this condition. The accompanying Rabi splitting can then be understood as simply normal mode hybridization on the nuclear-photonic potential energy surface, as already noted in the original article demonstrating vibrational strong coupling [84] and discussed in more detail in Appendix B.

In the context of cavity-modified chemical reactivity in the ground state, the formal equivalence between photonic and nuclear motion in the CBOA, in particular, allows us to apply standard tools such as transition state theory to obtain an estimation for reaction rates. TST implies that it should only be necessary to calculate the effective energy barrier for the reaction within the ground-state CBO surface.

We check the validity of TST for the model studied in Sec. III, i.e., the Shin-Metiu model coupled to a cavity mode on resonance with the first vibrational transition. The two-dimensional PES $\tilde{V}_{0}(R, q)$ is shown in Fig. 3(a) for a coupling strength of $\lambda=0.02$ a.u., which corresponds to a vibrational Rabi splitting of $\Omega_{R} \approx 0.05 \omega_{\nu}$. The second panel, Fig. 3(b), shows the minimum along $q$ of this surface as a function of $R$, i.e., along the path indicated by the curved dashed line in Fig. 3(a), for a set of coupling strengths $\lambda$ that induce a Rabi splitting of up to $\Omega_{R}=0.1 \omega_{\nu}$. This path closely corresponds to the minimum energy path of the proton transfer reaction within the CBOA. As the coupling is increased, the minima become deeper, while the transition state (TS) at $R=0$ stays unaffected. This leads to an effective increase of the
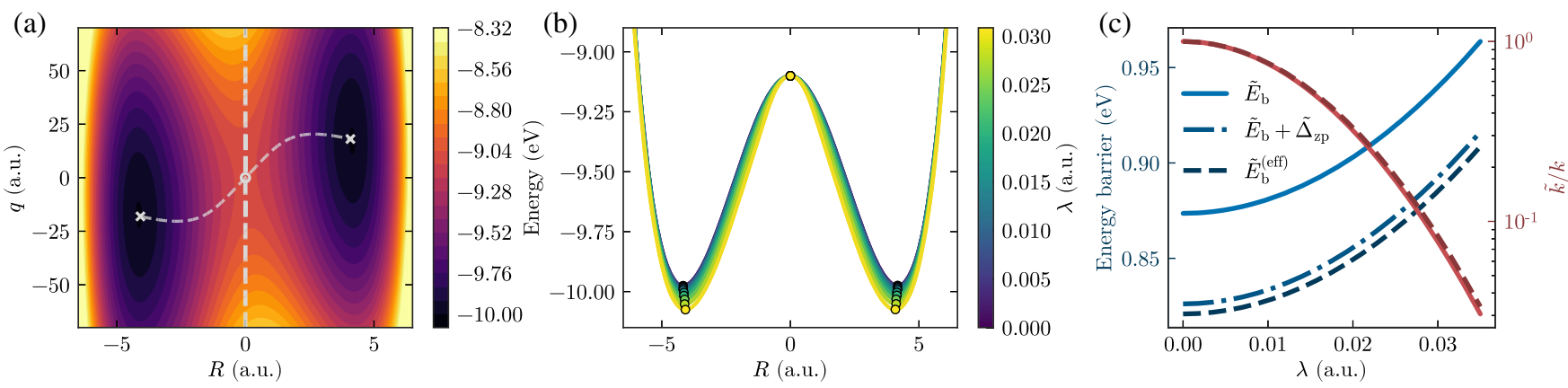

FIG. 3. (a) Two-dimensional ground-state PES in the cavity Born-Oppenheimer approximation for the Shin-Metiu model for $\lambda=$ 0.02 a.u. and $\omega_{c}=72.6 \mathrm{meV}$. At $R=0$, we show the dividing surface used to compute the reaction flux from reactant to product states. The gray dashed curve corresponds to the energy path along $q_{m}(R)$, i.e., the minimum in $q$. (b) Value of the energy path $V_{0}\left(\mathbf{R}, q_{m}\right)$ for different values of the Rabi frequency, which is related to the coupling strength through $\Omega_{R}=\lambda \mu_{0}^{\prime}\left(R_{0}\right)$, where the dipole derivative is evaluated at the minimum, as discussed in Appendix B. (c) Energy barrier and rate ratio versus the coupling strength for the case of a CBOA calculation (full lines) and for the effective energy barrier fitted from exact quantum rate calculations (dashed lines). 
reaction barrier $\tilde{E}_{b}=\tilde{V}_{0}\left(R_{\mathrm{TS}}, q_{\mathrm{TS}}\right)-\tilde{V}_{0}\left(R_{\min }, q_{\min }\right)$, as shown in Fig. 3(c). This panel also shows the corresponding change in the rate predicted by Eq. (8). The full lines correspond to the energy barrier calculated within the CBOA (blue) and the corresponding rate (red) according to TST, while the dashed lines show the effective energy barrier $E_{b}^{\text {(eff) }}$ extracted from the fit to the Arrhenius plot in Fig. 2 and the corresponding change in the rate obtained from the full quantum rate calculation in Sec. III. As can be seen, the effective and CBOA energy barriers agree very well, with just an approximately constant overestimation of the barrier in CBOA due to quantum effects such as zeropoint energy and tunneling. Consequently, the change of the reaction rate obtained from the full quantum calculation and the CBOA-TST prediction show excellent agreement. As expected from our previous discussion, the reaction rate of the hybrid cavity-molecule system decreases dramatically as the coupling increases due to the increase of the energy barrier height. Finally, we also calculate the CBOA energy barrier corrected by $\tilde{\Delta}_{\mathrm{zp}}$, the difference between the zero-point vibrational frequencies at the minimum and transition states as obtained from the Hessian of the PES (disregarding the direction of negative curvature at the TS). This corrected barrier is shown as a dash-dotted line in Fig. 3(c) and considerably improves the absolute agreement with the effective barrier extracted from the full quantum rate calculations.

While we up to now worked within a single-mode model, the CBO approximation actually makes it straightforward to treat multiple photonic modes. The ground-state PES then parametrically depends on multiple parameters $q_{k}$, one for each mode, just as a realistic molecule depends on multiple nuclear positions $\mathbf{R}_{i}$. Similarly, the adiabatic surfaces are not harder to calculate than for the single-mode case, and minimization strategies can rely on the same approaches used in "traditional" quantum chemistry. We note that, for a general cavity, the mode parameters can be obtained either by explicitly quantizing the modes (which is, in general, a difficult proposition) or, alternatively, by rewriting the spectral density of the light-matter coupling (proportional to the EM Green's function) as a sum of Lorentzians [77,86-88].

\section{PERTURBATION THEORY}

As we have seen, the cavity Born-Oppenheimer approximation provides a convenient picture to evaluate cavityinduced changes in chemical reactivity based on energy barriers in electronic PESs that are parametric in nuclear and photonic coordinates. In particular, the interaction term $\omega_{c} q \boldsymbol{\lambda} \cdot \hat{\boldsymbol{\mu}}$, with $q$ a parameter, is equivalent to that obtained from applying a constant external electric field. The CBO PES for arbitrary molecules can thus be calculated with standard quantum chemistry codes. However, obtaining the barrier, in general, still requires minimization of the molecular PES along the additional photon coordinate $q$ (or coordinates $q_{k}$, if multiple modes are treated). If the coupling is not too large and the relevant values of $q$ are small enough, the CBO ground-state PES can instead be obtained within perturbation theory, which up to second order in $\lambda$ is given by

$$
\tilde{V}_{0}(\mathbf{R}, q) \approx V_{0}(\mathbf{R})+\frac{\omega_{c}^{2}}{2} q^{2}+\lambda \omega_{c} q \mu_{0}(\mathbf{R})-\frac{\lambda^{2}}{2} \omega_{c}^{2} q^{2} \alpha_{0}(\mathbf{R}),
$$

where $V_{0}(\mathbf{R})$ and $\mu_{0}(\mathbf{R})$ are the bare-molecule groundstate PES and dipole moment, respectively, while $\alpha_{0}(\mathbf{R})$ is the ground-state static polarizability [89],

$$
\alpha_{0}(\mathbf{R} ; \omega=0)=2 \sum_{m \neq 0} \frac{\left|\mu_{m, 0}(\mathbf{R})\right|^{2}}{V_{m}(\mathbf{R})-V_{0}(\mathbf{R})}
$$

and encodes the effect of excited electronic levels, with $\mu_{m, 0}(\mathbf{R})$ the transition dipole moment between baremolecule electronic levels $m$ and 0 . Obtaining the full ground-state CBO surface within this approximation then requires just the calculation of the bare-molecule groundstate properties $V_{0}(\mathbf{R}), \mu_{0}(\mathbf{R})$, and $\alpha_{0}(\mathbf{R})$.

In addition to providing an explicit expression for the CBO ground-state PES in terms of bare-molecule groundstate properties, the simple analytical dependence on $q$ in Eq. (11) allows us to go one step further and obtain explicit expressions for the local minima and saddle points (i.e., transition states). In these configurations, the conditions $\partial_{q} \tilde{V}_{0}(\mathbf{R}, q)=\partial_{\mathbf{R}} \tilde{V}_{0}(\mathbf{R}, q)=0$ are satisfied. This yields a set of coupled equations that can be solved in order to find the configuration of the new critical points along the reaction path. The first equation gives the explicit condition

$$
q_{m}(\mathbf{R})=-\frac{\lambda}{\omega_{c}} \frac{\mu_{0}(\mathbf{R})}{1-\lambda^{2} \alpha_{0}(\mathbf{R})},
$$

which can be used to obtain the potential profile along the minimum in $q$ :

$$
\tilde{V}_{0}\left(\mathbf{R}, q_{m}\right)=V_{0}(\mathbf{R})-\frac{\lambda^{2}}{2} \mu_{0}^{2}(\mathbf{R})+\mathcal{O}\left(\lambda^{4}\right),
$$

where we drop terms of the order of $\lambda^{4}$, since the perturbation-theory PES [Eq. (11)] is accurate only to second order. This shows that the energy barrier on the CBO surface (within second-order perturbation theory) can be calculated directly from the bare-molecule potential and permanent dipole moment. In Fig. 4, we analyze the validity of Eq. (14) for computing the barrier height within the Shin-Metiu model. It can be observed that perturbation theory works quite well for the whole range of couplings, with a relative error in the cavity-induced change of the energy barrier of about $10 \%$ for the largest considered 


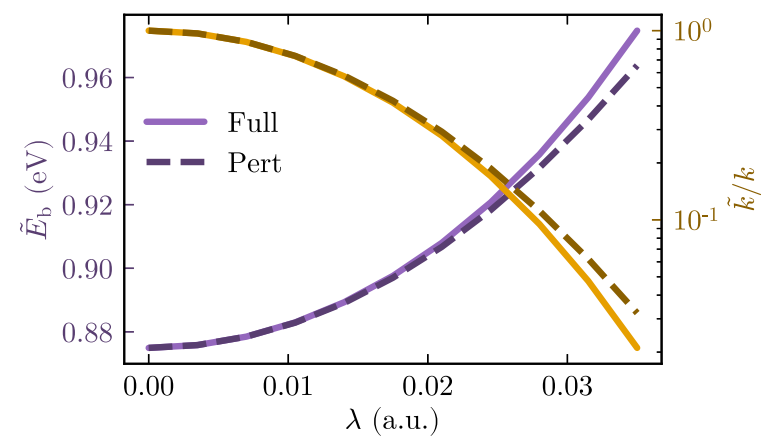

FIG. 4. Cavity Born-Oppenheimer energy barrier (purple lines) and relative change of reaction rates (yellow lines) for the ShinMetiu model inside a cavity, calculated to all orders in the lightmatter coupling strength $\lambda$ (solid lines) and up to second order in perturbation theory (dashed lines).

couplings. Because of the exponential dependence of the rates on the barrier height, this difference corresponds to an appreciable error in the rate constant but still provides a reasonable estimate. Note that, in the case of the ShinMetiu model, the error of the energy barrier stems entirely from the change at the minimum configuration, as the transition state has a zero dipole moment due to symmetry and is not affected by the cavity.

It is interesting to point out that Eq. (14) closely resembles the expression obtained in electric-field catalysis, where an external voltage is applied [90], or to electrostatic shifts provided by some catalysts [91]. This strategy exploits the Stark effect, i.e., the energy shift observed in the presence of a static electric field, to induce changes in the energies of transition state relative to the minimum configuration. As noted before, the CBOA corresponds to treating the influence of the cavity through an adiabatic parameter $q$ determining the electric field strength. However, instead of being externally imposed, in our case the effective field, determined by Eq. (13), is the one induced in the cavity by the permanent dipole moment of the molecule itself. This result also lends itself to an electrostatic interpretation of the effect.

In addition to the minimum energy barrier of the CBO PES itself, the effective energy barrier is also affected by the zero-point energy due to the quantization of nuclear and photonic motion (see Fig. 3). We can obtain its cavityinduced shift within perturbation theory by using Eq. (13) to rewrite Eq. (11) as

$$
\tilde{V}_{0}(\mathbf{R}, q)=\tilde{V}_{0}\left(\mathbf{R}, q_{m}\right)+\frac{\omega_{\text {eff }}^{2}(\mathbf{R})}{2}\left[q-q_{m}(\mathbf{R})\right]^{2},
$$

where $\omega_{\text {eff }}(\mathbf{R})=\omega_{c}-\left(\lambda^{2} / 2\right) \omega_{c} \alpha_{0}(\mathbf{R})+\mathcal{O}\left(\lambda^{4}\right)$, such that the photonic zero-point energy $\omega_{\text {eff }}(\mathbf{R}) / 2$ is decreased due to the polarizability of the molecule. We note that this expression accounts only for the quantization of the photonic motion along $q$. As we show in Appendix B, close to a local minimum at $R_{0}$, there is an additional correction due to the vibrational contribution to the molecular polarizability, which to second order is given by $-\omega_{c} \Omega_{R}^{2} /$ $\left[4 \omega_{v}\left(\omega_{c}+\omega_{v}\right)\right]$, where $\Omega_{R}=\lambda \mu_{g}^{\prime}\left(R_{0}\right) / \sqrt{M}$ is the onresonance vibrational Rabi splitting as discussed in Sec. II. As can be appreciated from Fig. 3, the contributions due to zero-point (photonic and vibrational) fluctuations contribute only negligibly to the change in the reaction rate in the Shin-Metiu model.

In general, a significant change of polarizability (either electronic or vibrational, which can be comparable in some molecules [92-94]) from the equilibrium to the transitionstate configuration could lead to similarly large effects as a change in the permanent dipole moment, especially if the cavity frequency $\omega_{c}$ is relatively large. However, it can be estimated that the vibrational contribution to the zeropoint energy shift is negligible for conditions typical for vibrational strong coupling. To be precise, at resonance $\omega_{c}=\omega_{v}$, this contribution reduces to $-\Omega_{R}^{2} /\left(8 \omega_{v}\right)$. Even for a relatively large vibropolariton Rabi splitting of $\Omega_{R} \approx 0.2 \omega_{v}$ [84,95,96], this contribution is of the order of approximately $10^{-2} \omega_{v}$ and, thus, small compared to typical barrier heights.

Finally, we note that the energy shifts above can be straightforwardly generalized to the case of multiple cavity modes within second-order perturbation theory. As can be easily verified, this simply leads to a sum over modes $k$, giving a final energy shift

$$
\delta E(\mathbf{R})=-\sum_{k} \frac{\lambda_{k}^{2}}{2}\left(\mu_{0}^{2}(\mathbf{R})+\frac{\omega_{k}}{2} \alpha_{0}(\mathbf{R})\right) .
$$

This general expression, which is just the second-order energy correction due to coupling to a set of cavity modes within the $\mathrm{CBO}$, corresponds to the well-known CasimirPolder energy shift [97]. The additional CBO approximation, in which nonadiabatic transitions between electronic surfaces are neglected, amounts to the approximation that the relevant cavity frequencies $\omega_{k}$ are much smaller than the electronic excitation energies $V_{m}(\mathbf{R})-V_{0}(\mathbf{R})$, such that only the (electronic) zero-frequency polarizability $\alpha_{0}(\mathbf{R})$ appears in the second term. In contrast, the first term depends only on the ground-state molecular permanent dipole moment $\mu_{0}=\langle 0|\hat{\mu}| 0\rangle$, which does not involve electronically excited states, and the CBOA thus does not amount to an additional approximation. In Appendix C, we demonstrate that, for the case that the cavity can be approximated as a point dipole (valid for a sufficiently small nanoparticle), the perturbative energy shifts obtained here correspond exactly to van der Waals forces [98], with the first term being the Debye force due to the interaction between the permanent molecular and the induced nanoparticle dipole and the second term the London force due to the interaction between fluctuating dipoles. Under the point-dipole approximation, the sum over cavity modes for 
the Debye force can furthermore be rewritten in terms of the zero-frequency polarizability of the nanoparticle.

Equation (16) is general for any kind of molecular reaction as long as the light-matter coupling is not too large. It demonstrates that the most relevant bare-molecule properties determining cavity-induced chemical reactions in the ground state are the permanent dipole moment and polarizability close to equilibrium, $\mu_{0}\left(\mathbf{R}_{0}\right)$ and $\alpha_{0}\left(\mathbf{R}_{0}\right)$, and transition-state, $\mu_{0}\left(\mathbf{R}_{\mathrm{TS}}\right)$ and $\alpha_{0}\left(\mathbf{R}_{\mathrm{TS}}\right)$, configurations and not the transition dipole moment of the vibrational excitation close to equilibrium, $\mu_{\nu} \propto \mu_{0}^{\prime}\left(\mathbf{R}_{0}\right)$, that determines the Rabi splitting. In addition to changing reaction barriers, it should be noted that the cavity-induced modification could potentially lead to a plethora of diverse chemical modifications, such as a change of the relative energy of different (meta)stable ground-state configurations and, thus, a change of the most stable configuration, or even the creation or disappearance of stable configurations. Furthermore, depending on the particular properties of the molecule, the cavity-induced change in the energy barriers can lead to either the suppression or acceleration of chemical reactions.

\section{MULTIMODE CAVITY: NANOPARTICLE ON MIRROR}

To demonstrate that the effects predicted above can be significant in realistic systems, we treat a nanoparticle-onmirror cavity with parameters taken from the experiment in Ref. [43], consisting of a spherical metallic nanoparticle (radius $R=20 \mathrm{~nm}$ ) separated by a small gap from a metallic plane; see the inset in Fig. 5. In this system, there is a series of multipole modes coupled to the molecule [77], with nontrivial behavior. Although several strategies can be employed to obtain the quantized light modes in this

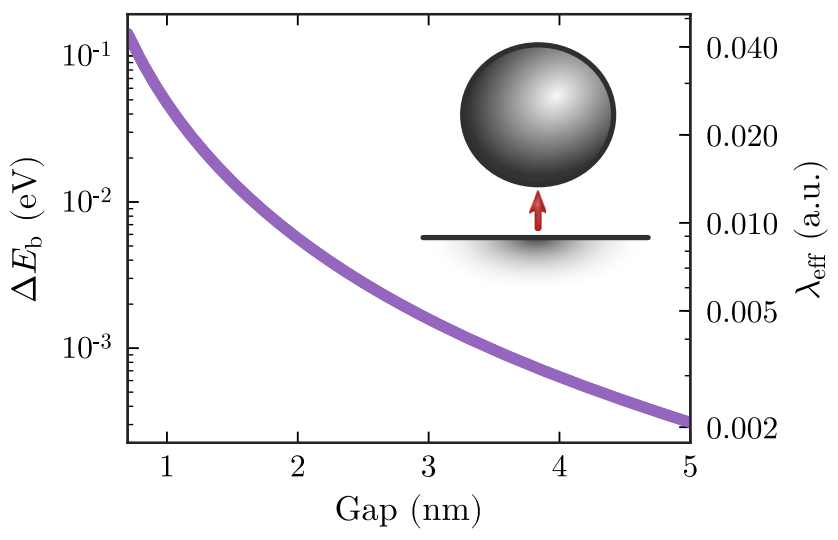

FIG. 5. Change of the energy barrier for the Shin-Metiu model inside a nanoparticle-on-mirror cavity as a function of the gap size. The right $y$ axis shows the corresponding values of the effective single-molecule coupling strength $\lambda_{\text {eff }}=\sqrt{\sum_{k} \lambda_{k}^{2}}$. Inset: Illustration of nanoparticle-on-mirror cavity geometry, with a single molecule placed in the nanogap between a planar metallic surface and a small metallic nanoparticle of radius $R=20 \mathrm{~nm}$. system [45,77], we instead exploit that the dominant contribution we found above is due to Debye-like electrostatic forces induced by the permanent molecular dipole and, thus, simply solve the electrostatic problem. To be precise, we calculate the energy shift of a permanent dipole in this cavity as obtained by its interaction with the field it induces in the cavity itself. Because of the simple involved geometric shapes (a sphere and a plane), this calculation can be achieved by the technique of image charges and dipoles (see Appendix D for details of the calculation). We furthermore rely again on perturbation theory; i.e., we assume that the molecular rearrangement due to its selfinduced field is negligible. Within this approximation, the energy shift we obtain from the purely electrostatic calculation is equivalent to the term proportional to $\mu_{0}^{2}$ in Eq. (16). The corresponding change $\Delta E_{b}$ in the height of the energy barrier for the Shin-Metiu molecule is shown in Fig. 5 as a function of the gap size (as a point of reference, the estimated gap size in Ref. [43] is $0.9 \mathrm{~nm}$ ). We find that the change in the energy barrier can be significant, corresponding to a change of the reaction rate by an order of magnitude or more (cf. Fig. 4). For comparison, in the figure we also show the effective coupling strength $\lambda_{\text {eff }}=\sqrt{\sum_{k} \lambda_{k}^{2}}$ corresponding to each gap size. This value corresponds to the coupling strength in a single-mode cavity that would give the same total energy shift as obtained in this realistic multimode cavity. We note that we here treat a perfectly spherical nanoparticle and do not include atomic-scale protrusions, which have been found to lead to even larger field confinement due to atomic-scale lightning-rod effects $[44,45,99]$. For the experimental gap size of $0.9 \mathrm{~nm}$, the effective coupling still becomes as large as $\lambda_{\text {eff }} \approx 0.031$ a.u., corresponding to $V_{\text {eff }}=4 \pi / \lambda_{\text {eff }}^{2} \approx$ $1.9 \mathrm{~nm}^{3}$. This result corresponds to a change in the energy barrier of $\delta E_{b} \approx 0.07 \mathrm{eV}$ for the Shin-Metiu model within second-order perturbation theory, which starts to break down at these couplings, as we previously saw in Fig. 4. This large effective coupling demonstrates the importance of the multimode nature of these cavities and the contribution of optically dark modes, as the "bright" nanogap plasmon mode that is seen in scattering spectra has an estimated mode volume of approximately $40 \mathrm{~nm}^{3}$.

\section{REALISTIC MOLECULE: 1,2-DICHLOROETHANE}

In the following, we apply the CBOA-TST to treat the internal rotation of 1,2-dichloroethane. In order to obtain the ground-state $\mathrm{CBO}$ surface under strong light-matter coupling, we calculate the (ground- and excited-state) baremolecule potential energy surfaces and permanent and transition dipole moments for a scan along the rotation angle (defined as the $\mathrm{Cl}-\mathrm{C}-\mathrm{C}-\mathrm{Cl}$ dihedral angle). For simplicity, we here use the relaxed ground-state configuration of the bare molecule for each rotation angle; i.e., we neglect cavity- 
induced changes in d.o.f. different from the internal rotation angle. The molecular properties are obtained with density functional theory calculations with the B3LYP [100] hybrid exchange-correlation functional and the $6-31+\mathrm{G}(\mathrm{d})$ basis set. Excited states are computed with time-dependent density functional theory within the Tamm-Dancoff approximation [101]. All calculations are performed with the TeraChem package $[102,103]$.

The rather simple 1,2-dichloroethane molecule presents several characteristic configurations along the rotation of the chlorine atoms around the axis defined by the carboncarbon bond (see the top in Fig. 6). It thus constitutes an excellent model system to show several possible effects induced by coupling to a cavity. In Fig. 6(a), we present the calculated ground-state energy landscape and dipole moment, while some relevant configurations are shown at the top. Analogously to the Shin-Metiu case, we present the path of minimum energy along $q$ in Fig. 6(b) but here calculated within perturbation theory [Eq. (14)]. We have explicitly checked that the contribution due to London forces is negligible here as well and focus on the Debyelike contribution in the following. We see that the most stable configuration $\left(\theta=180^{\circ}\right)$ shows no change due to the absence of a permanent dipole moment, while the most unstable one presents a large energy shift. Therefore, the different energy barriers of the system, represented versus the coupling strength in Fig. 6(c), are altered significantly. Here, we compare the energy barriers as predicted by perturbation theory (dashed lines) with the ones from a full diagonalization of the electronic Hamiltonian within the CBOA (full lines). In order to perform a full calculation, we calculate the electronic potential energy surfaces and the full dipole moment operator for a basis of 17 electronic states. We also indicate the points at which the coupling leads to important changes in the relative rates calculated with TST, i.e., the coupling or energy at which we achieve either suppression of $\tilde{k} / k=0.5$ or enhancement of $\tilde{k} / k=1.5$ or 2 . We see that in the case of perturbation theory (triangles) the energy changes are slightly underestimated, and, thus, larger couplings are needed to reach the same rate change as in the full calculation (circles).

As can be clearly seen, this still relatively simple molecule shows several different kinds of phenomena. We see that the reaction rate out of the global minimum at $\theta=180^{\circ}$, corresponding to $E_{3}$, is increased. On the other hand, $E_{1}$ increases, and the local minimum situated at $\theta=70^{\circ}$ is thus stabilized. Figure 6(b) suggests that this effect could potentially become more dramatic for larger couplings than treated here, as $\theta=70^{\circ}$ could become the new global minimum of the system. Finally, it is worth noting that the locations of the minima in energy also change for larger couplings. This shift is most noticeable for the minimum at $\theta=70^{\circ}$, which transforms to $\tilde{\theta} \approx 68^{\circ}$ for $\lambda=0.05$ a.u..

\section{RESONANCE EFFECTS}

The results presented above predict a change in the ground-state reactivity that is actually independent of the

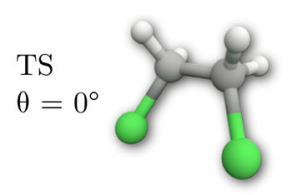

(a)



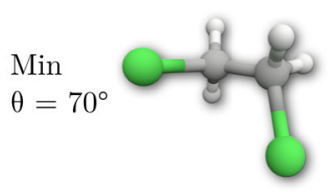

(b)

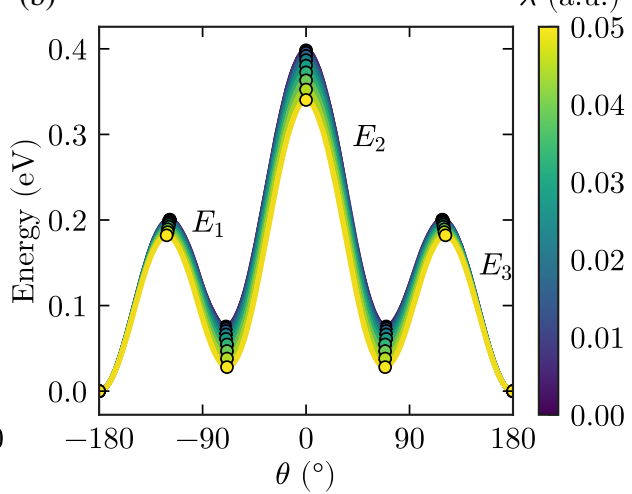

$\lambda($ a.u.) $\lambda($ a.u. $)$

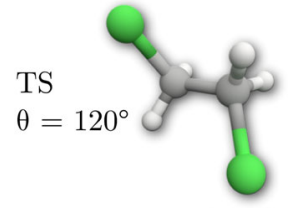

(c)



FIG. 6. Top: Different configurations along the internal rotation of 1,2-dichloroethane. (a) Energy landscape and dipole moment of the molecule. (b) Modified energy path for minimum $q$ for different coupling strengths. The energy barriers of the bare molecule are defined as $E_{1}=V\left(120^{\circ}\right)-V\left(70^{\circ}\right), E_{2}=V\left(0^{\circ}\right)-V\left(70^{\circ}\right)$, and $E_{3}=V\left(120^{\circ}\right)-V\left(180^{\circ}\right)$. (c) Relative modification of the energy barriers depending on the coupling strength for the full calculation (full lines, circles) and for perturbation theory (dashed lines, triangles). The marked points indicate relevant changes in the rate. 
cavity photon frequency and, in particular, does not rely on any resonance effects between the cavity mode and the vibrational transitions of the molecule. Although the $\mathrm{CBO}$ PES can and does represent vibropolariton formation through normal-mode hybridization, as discussed above and in Appendix B, the subsequent TST used to predict changes in chemical reaction rates is an inherently classical theory and does not depend on the quantized frequencies of motion on the PES nor, as mentioned above, on the transition dipole moment between vibrational levels (determined by the derivative of the permanent dipole moment). While we show that TST agrees almost perfectly with full quantum rate calculations, where nuclear and photonic motion is quantized and polariton formation is thus included, all calculations above were performed for the resonant case $\omega_{c}=\omega_{\nu}$.

We thus investigate whether there is any resonance effect on chemical ground-state reactivity by performing full quantum rate calculations for a wide range of cavity frequencies within the Shin-Metiu model. In Fig. 7, we represent the change $\tilde{k} / k$ in the calculated reaction rate of the coupled system relative to the uncoupled molecule as a function of $\omega_{c}$, for three different coupling strengths $\lambda$. Here, the values at $\omega_{c}=\omega_{\nu}$ correspond to the results shown in Fig. 3. We observe that the cavity rates are essentially constant with the frequency, with only a small modulation $\left[\tilde{k}\left(\omega_{c} \rightarrow \infty\right)-\tilde{k}\left(\omega_{c} \rightarrow 0\right) \neq 0\right]$ that becomes more important for larger couplings. For the cases represented in Fig. 7, this rate change goes from a relative modulation of $0.4 \%$ for $\lambda=0.005$ a.u. to a $7 \%$ modulation for $\lambda=0.02$ a.u.. However, no resonance effects are
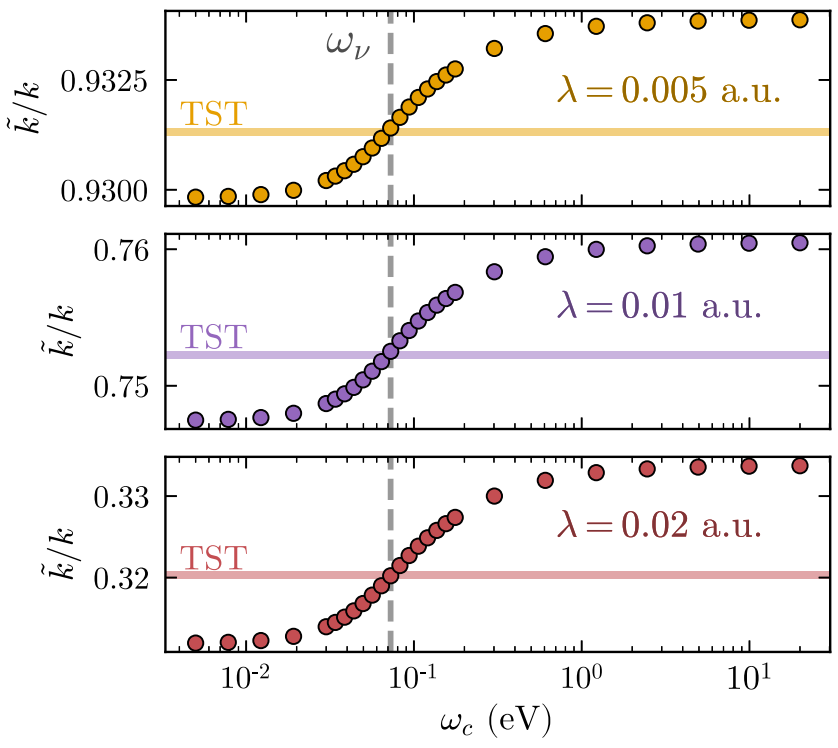

FIG. 7. Ratio between on- $(\tilde{k})$ and off-cavity $(k)$ rates vs the cavity frequency for three different values of the coupling strength. We increase the density of points close to the vibrational frequency of the molecule $\omega_{\nu} \approx 72.6 \mathrm{meV}$ in order to explore potential resonance effects. revealed close to the vibrational frequency of the molecule, $\omega_{\nu}$. At the same time, the vibrational frequency appears to be the relevant energy that separates the high- and lowfrequency limits for the rates, with TST working particularly well exactly around that value. In the following, we show that both limits can be understood by different additional adiabatic approximations.

In the high-frequency limit $\omega_{c} \gg \omega_{\nu}$, the photonic d.o.f. is fast compared to the vibrational one and can, thus, be assumed to instantaneously adapt to the current nuclear position $R$. This assumption implies that the photonic d.o.f. can be adiabatically separated (just like the electronic ones), and nuclear motion takes place along an effective 1D surface determined by the local minimum in $q$, i.e., along the path sketched in Fig. 3(a), or, within the lowestorder perturbation theory, along the surface defined by Eq. (14). Quantum rate calculations along this effective 1D PES indeed reproduce the reaction rate in the high-frequency limit perfectly (not shown). Furthermore, we note that, in this limit, it becomes convenient to directly group the photonic and electronic d.o.f. to obtain polaritonic PESs $[17,28]$ when performing the Born-Oppenheimer approximation, as successfully used for electronic strong coupling. In particular, this approach leads to exactly the same expression for the effective ground-state PES [17].

In the low-frequency limit $\omega_{c} \ll \omega_{\nu}$, on the other hand, the photonic motion is much slower than the vibrations and can also be adiabatically separated. The photons are now too slow to adjust their configuration, and $q$ can be assumed to stay constant during the reaction. The full quantum rate can then be obtained by performing a thermal average of independent 1D quantum rate calculations for each cut in $q$ of the two-dimensional surface $\tilde{V}(R, q)$. Here, the (normalized) thermal weight at each $q, \mathcal{P}(q)=\exp [-\langle E\rangle(q) /$ $\left.k_{B} T\right]$, is calculated by calculating the average thermal energy of the system $\langle E\rangle(q)$ for constant $q$. Again, this approximation agrees perfectly with the full quantum rate calculation for $\omega_{c} \rightarrow 0$ (not shown).

These results imply that, on the single-molecule level, the formation of vibropolaritons when $\omega_{c} \approx \omega_{\nu}$ is not actually required or even relevant for the cavity-induced change in ground-state chemical structure and reactivity. This fact can be appreciated by a simple intuitive argument: Vibrational strong coupling primarily occurs with the lowest vibrational transitions close to the equilibrium configuration, while chemical reactions that have to pass an appreciable barrier are typically determined by the properties of the involved transition state and the associated barrier height relative to the ground-state configuration. In general, neither of these are related to the properties of the lowest vibrational transitions (i.e., curvature of the PES and derivative of the dipole moment at the minimum).

The absence of resonance effects can also be appreciated through the connection to the well-known material-bodyinduced potentials obtained within perturbation theory. 
For example, if the EM mode is well approximated by a point-dipole mode, the obtained energy shift in the CBO PES can be rewritten as a van der Waals-like interaction between the permanent dipole moment of the molecule and the dipole it induces in the nanoparticle, which corresponds to the Debye force. In turn, the zero-point energy of the EM field reproduces the London dispersive force due to vacuum fluctuations and depends on the polarizability of the molecule. For an arbitrary EM environment, this effect can also be directly linked to Casimir-Polder forces [97,104], which exactly correspond to the generalization of emitter-emitter interactions to arbitrary material bodies (e.g., cavities). In particular, within the perturbative regime, the applicability of Casimir-Polder approaches could also be used to replace the explicit sum over modes $k$ by integrals involving the EM Green's function [47,73], which is readily available for arbitrary structures. This fact provides an additional argument for the absence of resonance effects in our calculations, as (ground-state) CasimirPolder forces are well known not to depend on resonances between light and matter d.o.f.

While we do not explicitly treat the situation in recent experiments on the modification of ground-state reactions by vibrational strong coupling (which are found to depend strongly on resonance conditions [14,35-37]), we believe that our results indicate that the resonance-dependent effects cannot be explained by a straightforward modification of ground-state reaction energy barriers at thermal equilibrium, as these would be captured by TST within the CBOA also in a many-mode, many-molecule setting.

\section{COLLECTIVE EFFECTS}

We now turn to the description of collective effects, i.e., the case of multiple molecules. For simplicity, we again restrict the discussion to a single cavity EM mode. As discussed in Sec. III, the single-molecule effects we investigated up to now become significant only for coupling strengths $\lambda=\sqrt{4 \pi / V_{\text {eff }}}$ corresponding to the smallest available plasmonic cavities, which typically operate at optical frequencies. However, typical experimental realizations of vibrational strong coupling are performed in micrometer-size cavities filled with a large number of molecules $[14,84,95,105]$. In this case, the per-molecule coupling $\lambda$ is so small that the single-molecule effects discussed above are completely negligible. For strong coupling and the associated formation of vibropolaritons, the coherent response of all molecules leads to a collective enhancement of the Rabi splitting $\Omega_{R, \text { col }}=\sqrt{N} \Omega_{R}$. However, as we have seen that the cavity-induced modification of the single-molecule ground state does not depend on the formation of polaritons, it is not a priori obvious whether this collective enhancement of the Rabi splitting also translates to cavity-induced collective modifications of the effective reaction barrier.
We thus repeat the analysis performed for the singlemolecule case above for the case of multiple molecules, working directly within the cavity Born-Oppenheimer approach. We note that the arguments for its applicability for treating ground-state chemical reactions translate straightforwardly from the single- to the many-molecule case. For $N$ identical molecules, the CBO light-matter interaction Hamiltonian becomes

$$
\begin{aligned}
\hat{H}_{e}^{(N)}= & \frac{\omega_{c}^{2}}{2} q^{2}+\sum_{i}\left[\hat{H}_{e}\left(\hat{\mathbf{x}}_{i} ; \mathbf{R}_{i}\right)+\omega_{c} q \boldsymbol{\lambda}_{i} \cdot \hat{\boldsymbol{\mu}}\left(\hat{\mathbf{x}}_{i} ; \mathbf{R}_{i}\right)\right] \\
& +\sum_{i, j} \hat{H}_{\mathrm{dd}}\left(\hat{\mathbf{x}}_{i}, \hat{\mathbf{x}}_{j} ; \mathbf{R}_{i}, \mathbf{R}_{j}\right),
\end{aligned}
$$

where $\hat{H}_{\mathrm{dd}}$ accounts for direct intermolecular (dipoledipole) interactions. We stress that we again assume that only a single cavity mode is significantly coupled to the molecules. The cavity-mediated dipole-dipole interaction is thus fully contained within the light-matter coupling term, and $\hat{H}_{\mathrm{dd}}$ corresponds to the free-space expression [66]. In the following discussion, we again use lowest-order perturbation theory to obtain analytical insight. The cavitymolecule and dipole-dipole interaction terms are then independent additive corrections. We first focus on the cavity-induced effects and discuss the influence of direct dipole-dipole interactions later, in particular, when studying a prototype implementation: a nanosphere surrounded by a collection of molecules. For simplicity of notation, we again use scalar quantities to indicate the component of the dipole along the field direction but keep the index $\epsilon$ to make this explicit, i.e., $\boldsymbol{\lambda}_{i}=\lambda_{i} \mathbf{\epsilon}_{i}$ and $\mathbf{\epsilon}_{i} \cdot \boldsymbol{\mu}\left(\mathbf{R}_{i}\right)=\mu_{\epsilon}\left(\mathbf{R}_{i}\right)$, so that we can rewrite the interaction term of the Hamiltonian as $\omega_{c} q \sum_{i}^{N} \lambda_{i} \hat{\mu}_{\epsilon}\left(\hat{\mathbf{x}}_{i} ; \mathbf{R}_{i}\right)$. The full Hamiltonian now corresponds to a many-body problem even for simple model molecules. Within second-order perturbation theory, the new (many-molecule) ground-state PES is

$$
\begin{aligned}
\tilde{V}_{0}^{(N)}\left(\mathbf{R}_{t}, q\right)= & \sum_{i} V_{0}\left(\mathbf{R}_{i}\right)+\frac{\omega_{c}^{2}}{2} q^{2}+\omega_{c} q \sum_{i} \lambda_{i} \mu_{0, \epsilon}\left(\mathbf{R}_{i}\right) \\
& -\frac{\omega_{c}^{2}}{2} q^{2} \sum_{i} \lambda_{i}^{2} \alpha_{0, \epsilon \epsilon}\left(\mathbf{R}_{i}\right)
\end{aligned}
$$

where $\mathbf{R}_{t}=\left(\mathbf{R}_{1}, \mathbf{R}_{2}, \ldots, \mathbf{R}_{N}\right)$ collects the nuclear configurations of all the molecules. With this result, we can again apply the corresponding conditions for finding critical points in order to analytically find the minimum along $q$ and the corresponding total energy of the hybrid system up to second order in $\lambda_{i}$ :

$\tilde{V}_{0}^{(N)}\left(\mathbf{R}_{t}, q_{m}\right)=\sum_{i} V_{0}\left(\mathbf{R}_{i}\right)-\frac{1}{2}\left(\sum_{i} \lambda_{i} \mu_{0, \epsilon}\left(\mathbf{R}_{i}\right)\right)^{2}$. 
It can be seen that the cavity-induced shift depends on the square of the sum of the (coupling-weighted) permanent dipole moments of the molecules, not on the sum of their squares. Assuming perfect alignment and identical configurations for all molecules, the energy shift becomes $-N^{2} \bar{\lambda}^{2}\left|\boldsymbol{\mu}_{0}(\mathbf{R})\right|^{2}$, where $\bar{\lambda}=(1 / N) \sum_{i} \lambda_{i}$ is the average coupling. The per-molecule energy shift is then linear in $N$, indicating a collective enhancement of the moleculecavity interaction. In contrast, the London-force-like change in the zero-point energy due to the modification of the effective cavity frequency is additive:

$$
\omega_{\mathrm{eff}}=\omega_{c}-\frac{\omega_{c}}{2} \sum_{i} \lambda_{i}^{2} \alpha_{0, \epsilon \epsilon}\left(\mathbf{R}_{i}\right)+\mathcal{O}\left(\lambda_{i}^{4}\right)
$$

with a total zero-point energy shift $\frac{1}{2}\left(\omega_{\text {eff }}-\omega_{c}\right)$ proportional to $N$, and shows no collective enhancement for singlemolecule reactions. It is interesting to note that the connection between polarizability and the dielectric function of a material through the Clausius-Mossotti relation suggests that this energy shift is equivalent to the change of mode frequency due to the refractive index of the collection of molecules. The shift in cavity mode frequencies due to refractive index changes after chemical reactions is exactly the effect used in experiments to monitor reaction rates under vibrational strong coupling [14,35,37]. We also mention that, at higher levels of perturbation theory, cavity-mediated contributions analogous to the Axilrod-Teller potential, i.e., van der Waals interactions between three emitters, appear in the intermolecular potential $[47,106]$.

Based on Eq. (19), we can analyze the effect of the cavity on the reaction rate of a single molecule within the ensemble. This rate is determined by the energy difference between minimum-energy and transition-state configurations of that molecule, with the other molecules fixed in a stable configuration (here chosen to be the minimum for all of them). For simplicity, we assume that the critical configurations $\mathbf{R}_{\mathrm{Min}}$ and $\mathbf{R}_{\mathrm{TS}}$ of the coupled system are equal to the uncoupled ones (as we saw above, the shifts are generally small). We can then directly express the change in the energy barrier of the moving molecule (chosen to be molecule $i=1$ here) as

$$
\begin{aligned}
\tilde{E}_{b}= & E_{b}-\frac{\lambda_{1}^{2}}{2}\left[\mu_{0, \epsilon}^{2}\left(\mathbf{R}_{1, \mathrm{TS}}\right)-\mu_{0, \epsilon}^{2}\left(\mathbf{R}_{1, \mathrm{Min}}\right)\right] \\
& -\lambda_{1}\left(\sum_{i=2}^{N} \lambda_{i} \mu_{0, \epsilon}\left(\mathbf{R}_{\mathrm{i}, \mathrm{Min}}\right)\right)\left[\mu_{0, \epsilon}\left(\mathbf{R}_{1, \mathrm{TS}}\right)-\mu_{0, \epsilon}\left(\mathbf{R}_{1, \mathrm{Min}}\right)\right] .
\end{aligned}
$$

This expression can be straightforwardly interpreted, with the first part corresponding to the Debye-like interaction of molecule 1 itself with the cavity and the second part corresponding to the cavity-mediated interaction of molecule 1 with all other molecules (which itself can be understood as the sum of two equal contributions, the interaction of the moving molecule with the cavity field induced by all other molecules, as well as the interaction of all other molecules with the cavity field induced by the molecule). Within perturbation theory, this Debye-like energy shift is again equivalent to the electrostatic energy, in this case that of a collection of permanent dipoles interacting with the cavity, i.e., a material structure. This equivalence makes the connection to electric field catalysis [90] even more direct, with the difference that the electric field is not generated by applying an external voltage but represents the cavity-enhanced field of all the other molecules. The fact that the main contribution is just the electrostatic energy shift also demonstrates the equivalence of our results to the approach of taking into account nonresonant effects through cavity-modified dipole-dipole and dipole-self interactions [66].

To treat the dependence on molecular orientations explicitly, we define the alignment angle $\theta_{i}$ for each molecule through $\mu_{0, \epsilon}\left(\mathbf{R}_{i}\right)=\left|\boldsymbol{\mu}_{0}\left(\mathbf{R}_{i}\right)\right| \cos \theta_{i}$. Inserting this expression in Eq. (21), we obtain

$$
\begin{aligned}
\tilde{E}_{b}= & E_{b}-\frac{\lambda_{1}^{2}}{2}\left[\mu_{0, \epsilon}^{2}\left(\mathbf{R}_{1, \mathrm{TS}}\right)-\mu_{0, \epsilon}^{2}\left(\mathbf{R}_{1, \mathrm{Min}}\right)\right] \\
& -N^{\prime} \bar{\lambda}^{2}\langle\cos \theta\rangle^{\prime}\left|\boldsymbol{\mu}_{0}\left(\mathbf{R}_{\mathrm{Min}}\right)\right| \\
& \times \lambda_{r, 1}\left[\mu_{0, \epsilon}\left(\mathbf{R}_{1, \mathrm{TS}}\right)-\mu_{0, \epsilon}\left(\mathbf{R}_{1, \mathrm{Min}}\right)\right],
\end{aligned}
$$

where $\lambda_{r, i}=\lambda_{i} / \bar{\lambda}$ is the relative coupling of molecule $i$, $\langle\cos \theta\rangle=(1 / N) \sum_{i} \lambda_{r, i} \cos \theta_{i}$ is the coupling-weighted average orientation angle, and primed quantities indicate that only molecules 2 to $N$ are taken into account (for $N \gg 1$, they can be replaced by unprimed quantities). We obtain a term proportional to the number of molecules $N$; i.e., there is a collective effect on the single-molecule energy barrier that is reminiscent of the collective Rabi splitting, $N \lambda^{2} \propto \Omega_{R, \text { col }}^{2}$. Note that the collective change of the energy barrier still depends on the molecule having a different permanent dipole moment in the transition and minimum configuration. Furthermore, it requires the molecules not participating in the reaction to have a nonzero permanent dipole moment and an average global alignment, such that $\langle\cos \theta\rangle \neq 0$. This condition could be fulfilled by fixing the molecular orientation by, e.g., growing self-assembled monolayers [107] or using DNA origami [50,108], or for molecules that can be grown in a crystalline phase, such as anthracene [109] (although polar molecules tend not to grow into crystals with a global alignment [110]). Another strategy to achieve alignment under strong coupling that has been successfully used experimentally is to align molecular liquid crystals through an applied static field [111]. However, for general disordered media such as polymers or molecules flowing in a liquid phase $[14,105]$, the angular distribution is typically isotropic, leading to $\langle\cos \theta\rangle \approx 0$. In that case, our theory 
predicts that no collective effect on reactivity should be observed unless the cavity itself induces molecular orientation (see below). We note for completeness that the collective Rabi splitting depends on the average of the squared $z$ component of the transition dipole moments, i.e., $\left\langle\cos ^{2} \theta\right\rangle$, which is nonzero unless all molecules are aligned perpendicular to the electric field of the cavity mode, and equal to $1 / 3$ for isotropic molecules.

In order to test the strength of the collective effect in realistic situations, and to compare it with the effect of direct (free-space) dipole-dipole interactions, we now treat a specific configuration, as depicted in Fig. 8(a): a nanocavity represented by a metallic sphere of diameter $d=8 \mathrm{~nm}$, surrounded by a collection of Shin-Metiu "molecules," located at distances from 1 to $16 \mathrm{~nm}$ from the sphere. We place a collection of up to $N=6000$ molecules at random positions within that volume, imposing a minimum distance of $1.5 \mathrm{~nm}$ between the molecules. A metal sphere with a Drude dielectric function (or a dielectric sphere with a single resonance, such as a phonon mode) can be approximated as a cavity with only three modes, the dipolar localized surface plasmon resonances aligned along $x, y$, and $z$ (see Appendix A for details). Higher-order multipole modes couple significantly only to emitters that are very close to the surface. We first assume all molecules to be aligned perfectly with the electric field of the z-oriented dipolar mode of the sphere. In this configuration, the sum over $x$ - and $y$-oriented fields at the origin cancels out for large $N$. For these directions, there is thus no Debye-like collective effect, and we can restrict our attention to just a single mode of the sphere (the $z$-oriented dipole mode). [112] As mentioned above, within perturbation theory, where the Debye-force-like contribution can be understood within a fully electrostatic picture, it is straightforward to include the direct (free-space) permanent-dipole-permanent-dipole interaction, as it is simply a further additive electrostatic contribution. In Fig. 8(b), we show the total electrostatic energy of the system, as well as the relative contributions due to molecule-sphere and direct molecule-molecule interactions, as a function of $N$. For the configuration considered here, for which we do not perform any optimization of total energy, the dipoledipole interactions give a positive contribution to the total energy that is significantly larger than the collective dipole-sphere interaction. The relative strength of dipoledipole and dipole-sphere interactions depends on the details of the configuration, and we have checked that, e.g., it is also possible to maintain the same collective interaction while obtaining an overall negative contribution from dipole-dipole interactions by not choosing random positions as we did for simplicity.

In contrast to the total energy, the change in the energy barrier predicted by Eq. (21) for the most strongly coupled molecule of the ensemble is dominated by the (collective) sphere-dipole interactions, as shown in Fig. 8(c). The barrier height indeed increases approximately linearly with $N$, with changes of up to approximately $0.09 \mathrm{eV}$ due to the cavity-mediated interaction and an associated suppression of the reaction rate by a factor of approximately 30 at room temperature. In the geometry treated here, the energy shift of the target molecule due to dipoledipole interactions with the other molecules also increases linearly with $N$, as the molecular dipoles combine to all act in the same direction at the sphere location, with an effect that is roughly half of the cavity-mediated interaction. As mentioned above, the details depend strongly on the configuration and cavity properties, and, in particular, it is also possible to choose configurations where the direct


FIG. 8. (a) Sketch of the model system of a collection of molecules distributed around a metal nanosphere with a diameter of 8 nm. The molecules are placed randomly at distances from 1 to $16 \mathrm{~nm}$ to the surface of the sphere, with the (permanent) dipoles aligned along the direction of the field of the sphere's $z$-oriented dipole mode. (b) Energy due to the dipole-sphere $\left(E_{\mathrm{ds}}\right)$ and dipole-dipole $\left(E_{\mathrm{dd}}\right)$ interactions in the system within perturbation theory as a function of the number of molecules $N$, as well as their sum $\left(E_{\text {tot }}\right)$. (c) Change in the energy barrier and corresponding change in the reaction rate at room temperature for the most strongly coupled molecule, also resolved into contributions from dipole-sphere and dipole-dipole interactions. In both (b) and (c), the slightly transparent lines correspond to different random realizations of the system, with the averages in solid lines. 
dipole-dipole interactions dominate. While a more exhaustive treatment is beyond the scope of this article, we mention that, in initial explorations, we do not find any simple configuration where the cavity-mediated interactions are significantly larger than direct dipole-dipole interactions.

While the barrier height increases here, the effect we predict can also lead to a decrease, for example, in the case that the transition-state dipole moment is larger than in the minimum configuration; cf. Eq. (22). Such a decrease would be expected, e.g., in dissociation reactions in which the molecule splits into two partially charged fragments and is also seen for the backreaction from the right to left minimum in the Shin-Metiu model for the case that all other molecules are in the leftmost minimum (see Fig. 9).

For comparison, Fig. 9 shows the effect of average alignment for the sphere-molecule system considered above, for the case of $N=6000$ molecules corresponding to a molecular density of approximately $2 \times 10^{8} \mu \mathrm{m}^{-3}$. It displays the CBO PES within second-order perturbation theory as a function of $R_{1}$, with all other molecules fixed in the minimum configuration, and along the photonic minimum $q=q_{m}$. For $\langle\cos \theta\rangle=1$, this result demonstrates that the collective cavity effect on the surface is significant, with the position of the critical points shifting compared to the bare molecule. For the Shin-Metiu model studied here, the barrier height is actually increased compared to the approximate prediction Eq. (21), which does not take into account these shifts. In contrast, when there is no average orientation, $\langle\cos \theta\rangle=0$, the effect on the surface is minimal and is reduced to the single-molecule result.

The single-molecule energy shifts we predict for perfect alignment can be significant, implying that the molecules, if they are free to rotate in place, could lower their energy by aligning with the electric field of the cavity mode, which could possibly lead to self-organization (for the example

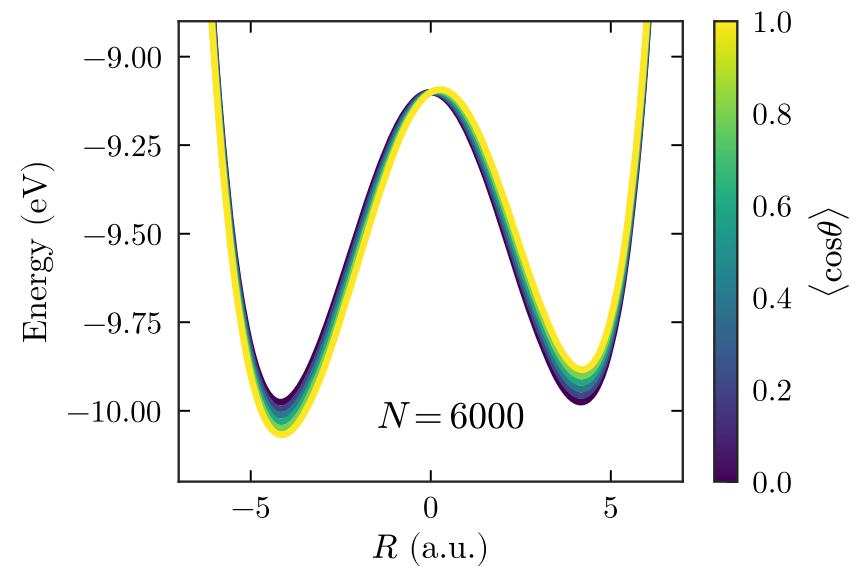

FIG. 9. Alignment dependence of the cavity Born-Oppenheimer PES along the photonic minimum path $q_{m}$ for the molecule in Fig. 8(c), with all other molecules fixed to the equilibrium position $R_{\min }$. system above, this process also requires breaking of the overall spherical symmetry). The details of this effect depend on the precise setup, such as the cavity material and shape, molecular and solvent properties, etc., and require a more complete treatment taking thermodynamical effects and free energy into account [113,114], which is beyond the scope of the current work. However, we mention that it has recently been shown that strong coupling and the associated formation of polaritons itself could lead to alignment due to the associated decrease of the lower polariton energy, provided that a significant fraction of molecules are excited to lower polariton states $[29,115]$. Although thermal excitation can be efficient for vibrational strong coupling due to the relatively low energies of vibropolaritons, on the order of a few times the thermal energy $k_{B} T$, it should be noted that the arguments in Refs. [29,115] do not directly translate to thermal-equilibrium situations. In that case, a change in the state energy due to improved orientation also leads to a change in the population, with the average energy per d.o.f. staying constant and, thus, no net energy gain.

Finally, we mention that the generalization of the above single-molecule arguments to the case of many cavity modes is not straightforward, and the results are thus not directly applicable to, e.g., Fabry-Perot cavities with a continuum of modes following a dispersion relation as a function of the in-plane wave vector, as employed in existing experiments [14,35-37]. Our results indicate that solving the electrostatic problem (where all modes are implicitly taken into account) should predict the changes in energy barriers, but, e.g., the scaling with the number of molecules is not immediately obvious, and, as mentioned above, statistical effects should be treated more carefully. Only for the special case that all modes have the same electric field distribution (e.g., different dipolar resonances of a small nanoparticle) can the sum over modes be performed straightforwardly.

\section{CONCLUSIONS}

In summary, we have analyzed modifications of groundstate chemical reactivity in hybrid cavity-molecule systems, motivated by experimental results showing this effect for vibrational strong coupling $[14,37]$. By treating a simple model system, the Shin-Metiu model, we were able to show through full quantum rate calculations on the singlemolecule level that ground-state thermally driven reaction rates can indeed be significantly modified under strong light-matter coupling. We then demonstrated that this change can be interpreted through classical transition-state theory, i.e., by the change in the height of an effective energy barrier (or activation energy) by working within the cavity Born-Oppenheimer approximation. In this approximation, the cavity photon is formally treated like a nucleus, such that ground-state reactions can be represented through motion on a PES with a single additional nuclearlike d.o.f. 
The use of perturbation theory leads to simple analytic expressions relating the effective barrier heights to purely ground-state molecular properties, namely, the uncoupled ground-state PES, dipole moment, and polarizability of the molecule. We showed that, within second-order perturbation theory, the energy shifts determining the barrier height on the CBO PES can be directly related to well-known intermolecular forces, i.e., the Debye and London forces, and more generally to Casimir-Polder interactions.

We stress that, while perturbation theory allows us to make connections to well-known results, our approach generalizes Casimir-Polder forces beyond the perturbative regime and applies for any coupling strength. Additionally, we have shown explicitly that the emergence of vibrational strong coupling does not affect the validity of the derived expressions for the effective energy barriers. At the same time, the CBOA provides a straightforward way to connect to well-known theories of chemical reactivity. The fact that the energy shifts obtained here become appreciable for realistic nanocavities with strongly subwavelength field confinement and, thus, sufficiently large $\lambda$ demonstrates that the (generalized) van der Waals forces due to the interaction of the molecular dipole with the polarization it induces in the cavity can become strong enough to lead to significant changes in chemical reactivity.

We also note that, in the context of Casimir-Polder forces, it is well known that, for subwavelength separations between emitters and material systems, it is sufficient to work within the quasistatic approximation, in which only the longitudinal electromagnetic Green's function contributes and the interaction does not depend on whether the Power-Zienau-Woolley transformation has been performed or not. In this context, it is also well known how to go beyond the quasistatic approximation, and the contribution from longitudinal and transversal fields (including the $\hat{\mathbf{A}}^{2}$ term and all EM-field modes) is naturally included within the Green's function [73].

We demonstrated the applicability of our approach for a realistic multimode cavity, a nanoparticle-on-mirror setup [43], and found that the effective single-molecule coupling strength in this case becomes significant (corresponding to a mode volume of approximately $2 \mathrm{~nm}^{3}$ ) even though the mode volume of the main optically active mode is significantly larger (approximately $40 \mathrm{~nm}^{3}$ ). We furthermore applied our theory to a real molecule, 1,2-dichloroethane, and showed that reaction rates can be both suppressed and enhanced depending on the relative value of the molecular dipole moment at the critical configurations (local minima and saddle points of the PES). A cavity could thus serve as a catalyst or as an inhibitor of a ground-state reaction and could even alter the global equilibrium configuration of the molecule, all without any kind of external energy input, with all reactions simply driven by thermal fluctuations. This result represents a potential way to efficiently optimize the desired yield of a molecular reaction.
We then found that, on the single-molecule level, the effects discussed above do not rely on any particular relation between the cavity photon frequency $\omega_{c}$ and the vibrational transitions in the molecule $\omega_{\nu}$ and, thus, in particular, not on the formation of polaritons (hybrid light-matter states). This finding is consistent with the interpretation of the energy shifts as generalizations of Casimir-Polder interactions beyond the perturbative regime. We also showed that the small modulation of the reaction rate as a function of $\omega_{c}$ that is observed numerically can be understood by simple adiabatic approximations and, again, is not related to polariton formation.

For the case of many-molecule strong coupling, where the single-molecule coupling $\lambda$ is typically so small that the single-molecule effects described above are negligible, we demonstrated that the PES and reaction barriers can be significantly modified by collective effects provided that the permanent dipole moments of the molecules are oriented with respect to the cavity-mode field, such that they induce an overall static electric field. However, it should also be noted that similar effects could be achieved by direct dipole-dipole interactions if one manages to align all molecules such as to create a strong field at the position of a single molecule. An interesting open question is whether the cavity-mediated interactions could induce alignment in materials that do not show it in the absence of the cavity, or if direct dipole-dipole interactions would prevent this alignment.

Finally, it should be noted that we have throughout assumed that the whole system is in thermal equilibrium, i.e., that the effective temperature is identical for both the molecules and the cavity EM mode. This assumption implies that system-bath interactions do not have to be explicitly modeled, as the system can simply be assumed to be at a given temperature (as explicitly included in the quantum rate calculations and TST). This assumption would break down if the internal vibrational temperature of the molecules is different from the temperature of the thermal radiation bath that the cavity is coupled to. In that case, the effective temperature of the system could potentially become an average of the internal and external bath temperatures. In particular, the effective temperature relevant for a given reaction could depend on whether vibrational motion along that reaction coordinate is hybridized with the cavity mode, such that the external blackbody radiation bath would conceivably couple more efficiently to that mode than to others. Such effects have been studied for Casimir-Polder forces, where resonant contributions that exactly cancel at thermal equilibrium can become important in nonequilibrium situations [116,117], and possibly give rise to additional collective effects [118].

Our work demonstrates the possibility of modifying ground-state chemical reactions and molecular properties in hybrid cavity-molecule systems without an external input of energy. We believe that the theory presented here lays the 
groundwork for a profound understanding of this novel cavity effect and could be used to predict experimentally available chemical modifications.

\section{ACKNOWLEDGMENTS}

We are grateful to M. Ruggenthaler, S. De Liberato, and P. Rabl for helpful discussions. This work has been funded by the European Research Council (ERC-2016STG-714870) and the Spanish MINECO under Contract No. MAT2014-53432-C5-5-R and the "María de Maeztu" program for Units of Excellence in R\&D (MDM-20140377), as well as through a Ramón y Cajal grant (J. F.).

Note added in proof.-We have recently shown how the effects discussed here can lead to self-induced catalysis for several common chemical reactions, and can also modify the transition temperature of spin-crossover complexes [119].

\section{APPENDIX A: QUANTIZED MODES OF SPHERICAL NANOPARTICLES}

We here show that, for two general models for the dielectric function, a spherical nanoparticle in a vacuum within the quasistatic approximation can be approximated as a three-mode cavity [120,121], with the modes corresponding to three degenerate dipole modes. We treat the sphere as a point dipole, equivalent to neglecting the short-range higher-order multipole modes that couple only to emitters very close to the surface [87,122]. The direction-independent polarizability of the sphere is then given by [123]

$$
\alpha_{S}(\omega)=a^{3} \frac{\epsilon(\omega)-1}{\epsilon(\omega)+2},
$$

where $a$ is the radius of the sphere. For a metallic Drude model dielectric function without losses, $\epsilon_{m}(\omega)=$ $1-\omega_{p}^{2} / \omega^{2}$, the polarizability can be rewritten as

$$
\alpha_{S}(\omega)=\frac{a^{3} \omega_{0}^{2}}{\omega_{0}^{2}-\omega^{2}},
$$

where $\omega_{0}=\omega_{p} / \sqrt{3}$. This result is identical to the polarizability of a single-mode quantum oscillator at frequency $\omega_{0}$ with transition dipole moment $\mu_{e g}=\sqrt{\omega_{0} a^{3} / 2}$ [89]:

$$
\alpha_{q}(\omega)=\mu_{e g}^{2}\left(\frac{1}{\omega_{0}-\omega}+\frac{1}{\omega_{0}+\omega}\right)=\frac{a^{3} \omega_{0}^{2}}{\omega_{0}^{2}-\omega^{2}} .
$$

Here, spherical symmetry implies that there are three degenerate quantum oscillators, corresponding to the quantized localized surface plasmon resonances in this case, directed along three orthogonal axes (e.g., $x, y$, and $z$ ). If the dielectric function is instead given by a Lorentzian function representing a material resonance (e.g., a phonon mode) at frequency $\omega_{\mathrm{ph}}$ and with a resonator strength characterized by $\omega_{f}$, i.e., $\epsilon(\omega)=1+\left[\omega_{f}^{2} /\left(\omega_{\mathrm{ph}}^{2}-\omega^{2}\right)\right]$, we again get the same form of the polarizability by using $\omega_{0}^{2}=$ $\omega_{\mathrm{ph}}^{2}+\left(\omega_{f}^{2} / 3\right)$ and $\mu_{e g}=\omega_{f} \sqrt{a^{3} /\left(6 \omega_{0}\right)}$, with the quantized mode now corresponding to a localized surface phonon polariton resonance. We thus find that these simple models can be quantized by considering just a single or a few cavity modes.

\section{APPENDIX B: NORMAL MODES IN CAVITY BORN-OPPENHEIMER}

In this section, we demonstrate how vibropolariton formation can be observed within the cavity BornOppenheimer approximation [25]. We note that this derivation is essentially identical to that performed in Ref. [84], although it was not based on the CBOA there. Within this approximation, discussed in detail in Sec. IV, the photonic d.o.f. is described by a continuous parameter $q$, proportional to the electric displacement field, and both nuclear and photonic motion takes places on an electronic potential energy surface parametric in $\mathbf{R}$ and $q$. Hybridization of photonic and vibrational excitations is thus not directly observed by inspecting the PES but requires calculating the coupled nuclear-photonic eigenstates determined by the PES. This calculation is most easily achieved close to a local minimum, where the surface can be locally approximated through coupled harmonic oscillator potentials and direct diagonalization of the Hessian gives the polariton eigenstates. Close to the minimum and treating only a single nuclear d.o.f. for simplicity, the $\mathrm{CBO}$ ground-state surface of Eq. (11) may be written as

$$
V_{0}(R, q)=\frac{\omega_{\nu}^{2}}{2} R^{2}+\frac{\omega_{c}^{2}}{2} q^{2}+\lambda \omega_{c} q \mu(R),
$$

where we here use mass-weighted coordinates $(R \rightarrow R / \sqrt{M})$ for the molecular coordinate, which has a vibrational frequency of $\omega_{\nu}$. For simplicity, we ignore the polarizability term, which is equivalent to redefining the effective photon frequency with the replacement $\frac{1}{2} \omega_{c}^{2}\left[1-\lambda^{2} \alpha(R)\right] q^{2} \rightarrow$ $\frac{1}{2} \omega_{c}^{2} q^{2}$. The Hessian of the surface is then

$$
\mathcal{H}=\left(\begin{array}{cc}
\omega_{\nu}^{2} & \lambda \omega_{c} \mu_{0}^{\prime}\left(R_{0}\right) \\
\lambda \omega_{c} \mu_{0}^{\prime}\left(R_{0}\right) & \omega_{c}^{2}
\end{array}\right),
$$

where $\mu_{0}^{\prime}\left(R_{0}\right)$ is the derivative of the ground-state dipole moment in mass-weighted coordinates, evaluated at the minimum. The eigenvalues of the Hessian correspond to the squares of the normal mode frequencies. In the resonant case $\left(\omega_{\nu}=\omega_{c}\right)$, it is straightforward to show that the new frequencies are $\omega_{ \pm}=\omega_{c} \sqrt{1 \pm\left(\lambda / \omega_{c}\right) \mu_{0}^{\prime}\left(R_{0}\right)}$, which is the standard result for the modes of two coupled harmonic 
oscillators beyond the rotating wave approximation [124]. The connection between the coupling strength and the Rabi splitting is clearer in the limit of low coupling:

$$
\omega_{ \pm} \approx \omega_{c} \pm \frac{1}{2} \lambda \mu_{0}^{\prime}\left(R_{0}\right)
$$

The Rabi splitting to lowest order is then $\Omega_{R}=\omega_{+}-\omega_{-}=$ $\lambda \mu_{0}^{\prime}\left(R_{0}\right)$, i.e., proportional to $\lambda$, which is the definition that we use in the main text. The Rabi splitting for collective strong coupling is enhanced as $\Omega_{R}=\sqrt{N} \lambda \mu_{0}^{\prime}\left(R_{0}\right)$.

With the results above, it is trivial to obtain the zeropoint energy in the coupled system, which is given by $\left(\omega_{+}+\omega_{-}\right) / 2$. To second order in $\lambda$, we obtain (without assuming resonance)

$$
E_{z p}=\frac{1}{2}\left(\omega_{c}+\omega_{v}\right)-\frac{\omega_{c} \lambda^{2} \mu_{0}^{\prime 2}\left(R_{0}\right)}{4 \omega_{v}\left(\omega_{c}+\omega_{v}\right)} .
$$

We note that, when the effective frequency $\omega_{\text {eff }}$ instead of the bare frequency $\omega_{c}$ is used for the cavity mode, the additional correction $-\left(\lambda^{2} / 4\right) \omega_{c} \alpha\left(R_{0}\right)$ corresponds to an additive term up to second order.

\section{APPENDIX C: NANOPARTICLE VAN DER WAALS POTENTIAL}

We here show that, for a general nanoparticle with a series of (bosonic) dipole resonances characterized by (vectorial) transition dipoles $\boldsymbol{\mu}_{k}$ and frequencies $\omega_{k}$, the perturbative energy shift due to coupling to a molecule [Eq. (16)] corresponds exactly to Debye and London forces. In this case, the coupling operators $\lambda_{k}$ at the molecular position $\mathbf{r}_{m}$ are determined by the static dipole-dipole interaction:

$$
\lambda_{k}=\sqrt{\frac{2}{\omega_{k}}}\left(\frac{3\left(\boldsymbol{\mu}_{k} \cdot \mathbf{r}_{m}\right) \mathbf{r}_{m}}{r_{m}^{5}}-\frac{\boldsymbol{\mu}_{k}}{r_{m}^{3}}\right) .
$$

For simplicity, we assume $\mathbf{r}_{m}$ to be along the $x$ axis and all dipoles to be oriented along $z$, leading to

$$
\lambda_{k}=\sqrt{\frac{2}{\omega_{k}}} \frac{\mu_{k}}{r_{m}^{3}} .
$$

Inserting this result into the perturbative energy correction then gives

$$
\begin{aligned}
\delta E(\mathbf{R}) & =-\sum_{k} \frac{\lambda_{k}^{2}}{2}\left(\mu_{0}^{2}(\mathbf{R})+\frac{\omega_{k}}{2} \alpha_{0}(\mathbf{R})\right) \\
& =-\sum_{k} \frac{\mu_{k}^{2} \mu_{0}^{2}(\mathbf{R})}{\omega_{k} r_{m}^{6}}-\sum_{k} \frac{\mu_{k}^{2} \alpha_{0}(\mathbf{R})}{2 r_{m}^{6}} .
\end{aligned}
$$

Using Eq. (A3), the sum in the first term can be replaced with the zero-frequency polarizability of the nanoparticle, $\alpha_{n}(0)=\sum_{k} 2 \mu_{k}^{2} / \omega_{k}$, giving

$$
\delta E(\mathbf{R})=-\frac{\alpha_{q}(0) \mu_{0}^{2}(\mathbf{R})}{2 r_{m}^{6}}-\sum_{k} \frac{\mu_{k}^{2} \alpha_{0}(\mathbf{R})}{2 r_{m}^{6}}
$$

where the first term corresponds exactly to the static energy of a dipole $\mu_{0}$ at $\mathbf{r}_{m}$ interacting with a polarizable sphere at the origin and the second term corresponds to the London force [125].

\section{APPENDIX D: ELECTROSTATICS OF A NANOPARTICLE-ON-MIRROR CAVITY}

We here derive the electrostatic energy of a dipole $\boldsymbol{\mu}$ inside a plasmonic nanocavity made up of a spherical metallic nanoparticle of radius $R$ separated by a gap $\Delta$ from a planar metallic mirror. This derivation can be achieved using the method of image charges by considering a formally infinite series of images, with each image in a component of the cavity inducing an image in the other. In practice, this infinite converging series can be truncated after a finite number of terms to obtain any desired degree of accuracy. Considering both a charge $q$ and a dipole $\boldsymbol{\mu}$ at position $\mathbf{r}$ relative to the center of a perfectly conducting grounded sphere of radius $R$, the resulting images are located at $\mathbf{r}^{\prime}=(R / r)^{2} \mathbf{r}$ (where $r=|\mathbf{r}|)$ and consist of a charge and dipole given, respectively, by

$$
\begin{gathered}
q^{\prime}=-\frac{R}{r} q+\frac{R}{r^{3}} \mathbf{r} \cdot \boldsymbol{\mu}, \\
\boldsymbol{\mu}^{\prime}=\left(\frac{R}{r}\right)^{3}\left[\frac{2 \mathbf{r}(\mathbf{r} \cdot \boldsymbol{\mu})}{r^{2}}-\boldsymbol{\mu}\right] .
\end{gathered}
$$

Here, it is important to take into account that the image of a dipole in a sphere always consists of both a charge and a dipole. The corresponding expressions for a plane can be obtained by simply taking $R \rightarrow \infty$ (and moving the center of the sphere accordingly to keep the planar surface fixed). The cavity-induced energy shift of the dipole is then given by $U=-\frac{1}{2} \mathbf{E}_{\text {ind }} \cdot \boldsymbol{\mu}$, where $\mathbf{E}_{\text {ind }}$ is the total field generated by all image dipoles and charges and the factor $\frac{1}{2}$ is due to them being induced.

It is also interesting to note that, since a dipole induces a nonzero image charge, the total induced dipole moment of the sphere is not origin independent. In particular, the induced dipole moment (for $q=0$ ) relative to the sphere center is $\boldsymbol{\mu}^{\prime}+\mathbf{r}^{\prime} q^{\prime}=(R / r)^{3}\left[3 \mathbf{r}(\mathbf{r} \cdot \boldsymbol{\mu}) / r^{2}-\boldsymbol{\mu}\right]$, which corresponds to the dipole moment obtained when treating the nanoparticle as a polarizable point particle (cf. Appendix A). Accordingly, in a multipole expansion about the sphere center, higher-order multipoles are nonzero, and neglecting them corresponds to an approximation, while using the image dipoles and charges as given above is exact. 
[1] R. Miller, T. E. Northup, K. M. Birnbaum, A. Boca, A. D. Boozer, and H. J. Kimble, Trapped Atoms in Cavity QED: Coupling Quantized Light and Matter, J. Phys. B 38, S551 (2005).

[2] H. Walther, B. T. H. Varcoe, B.-G. Englert, and T. Becker, Cavity Quantum Electrodynamics, Rep. Prog. Phys. 69, 1325 (2006).

[3] R. J. Thompson, G. Rempe, and H. J. Kimble, Observation of Normal-Mode Splitting for an Atom in an Optical Cavity, Phys. Rev. Lett. 68, 1132 (1992).

[4] D. Sanvitto and S. Kéna-Cohen, The Road towards Polaritonic Devices, Nat. Mater. 15, 1061 (2016).

[5] F. Barachati, S. De Liberato, and S. Kéna-Cohen, Generation of Rabi-Frequency Radiation Using ExcitonPolaritons, Phys. Rev. A 92, 033828 (2015).

[6] J. A. Ćwik, P. Kirton, S. De Liberato, and J. Keeling, Excitonic Spectral Features in Strongly Coupled Organic Polaritons, Phys. Rev. A 93, 033840 (2016).

[7] M. A. Zeb, P. G. Kirton, and J. Keeling, Exact States and Spectra of Vibrationally Dressed Polaritons, ACS Photonics 5, 249 (2018).

[8] E. Orgiu, J. George, J. A. Hutchison, E. Devaux, J. F. Dayen, B. Doudin, F. Stellacci, C. Genet, J. Schachenmayer, C. Genes, G. Pupillo, P. Samorì, and T. W. Ebbesen, Conductivity in Organic Semiconductors Hybridized with the Vacuum Field, Nat. Mater. 14, 1123 (2015).

[9] J. Feist and F. J. Garcia-Vidal, Extraordinary Exciton Conductance Induced by Strong Coupling, Phys. Rev. Lett. 114, 196402 (2015).

[10] X. Zhong, T. Chervy, L. Zhang, A. Thomas, J. George, C. Genet, J. A. Hutchison, and T. W. Ebbesen, Energy Transfer between Spatially Separated Entangled Molecules, Angew. Chem., Int. Ed. 56, 9034 (2017).

[11] F. J. Garcia-Vidal and J. Feist, Long-Distance Operator for Energy Transfer, Science 357, 1357 (2017).

[12] R. Sáez-Blázquez, J. Feist, A. I. Fernández-Domínguez, and F. J. García-Vidal, Organic Polaritons Enable Local Vibrations to Drive Long-Range Energy Transfer, Phys. Rev. B 97, 241407(R) (2018).

[13] J. A. Hutchison, T. Schwartz, C. Genet, E. Devaux, and T. W. Ebbesen, Modifying Chemical Landscapes by Coupling to Vacuum Fields, Angew. Chem. 124, 1624 (2012).

[14] A. Thomas, J. George, A. Shalabney, M. Dryzhakov, S. J. Varma, J. Moran, T. Chervy, X. Zhong, E. Devaux, C. Genet, J. A. Hutchison, and T. W. Ebbesen, Ground-State Chemical Reactivity under Vibrational Coupling to the Vacuum Electromagnetic Field, Angew. Chem., Int. Ed. 55, 11462 (2016).

[15] B. Munkhbat, M. Wersäll, D. G. Baranov, T. J. Antosiewicz, and T. Shegai, Suppression of Photo-Oxidation of Organic Chromophores by Strong Coupling to Plasmonic Nanoantennas, Sci. Adv. 4, eaas9552 (2018).

[16] I. V. Tokatly, Time-Dependent Density Functional Theory for Many-Electron Systems Interacting with Cavity Photons, Phys. Rev. Lett. 110, 233001 (2013).

[17] J. Galego, F. J. Garcia-Vidal, and J. Feist, Cavity-Induced Modifications of Molecular Structure in the StrongCoupling Regime, Phys. Rev. X 5, 041022 (2015).

[18] J. Flick, M. Ruggenthaler, H. Appel, and A. Rubio, Kohn-Sham Approach to Quantum Electrodynamical
Density-Functional Theory: Exact Time-Dependent Effective Potentials in Real Space, Proc. Natl. Acad. Sci. U.S.A. 112, 15285 (2015).

[19] J. Galego, F. J. Garcia-Vidal, and J. Feist, Suppressing Photochemical Reactions with Quantized Light Fields, Nat. Commun. 7, 13841 (2016).

[20] F. Herrera and F. C. Spano, Cavity-Controlled Chemistry in Molecular Ensembles, Phys. Rev. Lett. 116, 238301 (2016).

[21] M. Kowalewski, K. Bennett, and S. Mukamel, Cavity Femtochemistry: Manipulating Nonadiabatic Dynamics at Avoided Crossings, J. Phys. Chem. Lett. 7, 2050 (2016).

[22] M. Kowalewski, K. Bennett, and S. Mukamel, NonAdiabatic Dynamics of Molecules in Optical Cavities, J. Chem. Phys. 144, 054309 (2016).

[23] J. Galego, F. J. Garcia-Vidal, and J. Feist, Many-Molecule Reaction Triggered by a Single Photon in Polaritonic Chemistry, Phys. Rev. Lett. 119, 136001 (2017).

[24] L. A. Martínez-Martínez, M. Du, R. F. Ribeiro, S. KénaCohen, and J. Yuen-Zhou, Polariton-Assisted Singlet Fission in Acene Aggregates, J. Phys. Chem. Lett. 9, 1951 (2018).

[25] J. Flick, M. Ruggenthaler, H. Appel, and A. Rubio, Atoms and Molecules in Cavities, from Weak to Strong Coupling in Quantum-Electrodynamics (QED) Chemistry, Proc. Natl. Acad. Sci. U.S.A. 114, 3026 (2017).

[26] J. Flick, H. Appel, M. Ruggenthaler, and A. Rubio, Cavity Born-Oppenheimer Approximation for Correlated Electron-Nuclear-Photon Systems, J. Chem. Theory Comput. 13, 1616 (2017).

[27] H. L. Luk, J. Feist, J. Jussi Toppari, and G. Groenhof, Multiscale Molecular Dynamics Simulations of Polaritonic Chemistry, J. Chem. Theory Comput. 13, 4324 (2017).

[28] J. Feist, J. Galego, and F. J. Garcia-Vidal, Polaritonic Chemistry with Organic Molecules, ACS Photonics 5, 205 (2018).

[29] J. Keeling and P. G. Kirton, Orientational Alignment in Cavity Quantum Electrodynamics, Phys. Rev. A 97, 053836 (2018).

[30] L. A. Martínez-Martínez, R. F. Ribeiro, J. CamposGonzález-Angulo, and J. Yuen-Zhou, Can Ultrastrong Coupling Change Ground-State Chemical Reactions?, ACS Photonics 5, 167 (2018).

[31] M. Ruggenthaler, N. Tancogne-Dejean, J. Flick, H. Appel, and A. Rubio, From a Quantum-Electrodynamical LightMatter Description to Novel Spectroscopies, Nat. Rev. Chem. 2, 0118 (2018).

[32] R. F. Ribeiro, L. A. Martínez-Martínez, M. Du, J. CamposGonzalez-Angulo, and J. Yuen-Zhou, Polariton Chemistry: Controlling Molecular Dynamics with Optical Cavities, Chem. Sci. 9, 6325 (2018).

[33] O. Vendrell, Coherent Dynamics in Cavity Femtochemistry: Application of the Multi-Configuration TimeDependent Hartree Method, Chem. Phys. 509, 55 (2018).

[34] O. Vendrell, Collective Jahn-Teller Interactions through Light-Matter Coupling in a Cavity, Phys. Rev. Lett. 121, 253001 (2018).

[35] H. Hiura, A. Shalabney, and J. George, Cavity CatalysisAccelerating Reactions under Vibrational Strong Coupling, ChemRxiv 7234721. 
[36] J. Lather, P. Bhatt, A. Thomas, T. W. Ebbesen, and J. George, Cavity Catalysis by Co-Operative Vibrational Strong Coupling of Reactant and Solvent Molecules, ChemRxiv 7531544.

[37] A. Thomas, L. Lethuillier-Karl, K. Nagarajan, R. M. A. Vergauwe, J. George, T. Chervy, A. Shalabney, E. Devaux, C. Genet, J. Moran, and T. W. Ebbesen, Tilting a GroundState Reactivity Landscape by Vibrational Strong Coupling, Science 363, 615 (2019).

[38] T. Yamamoto, Quantum Statistical Mechanical Theory of the Rate of Exchange Chemical Reactions in the Gas Phase, J. Chem. Phys. 33, 281 (1960).

[39] W.H. Miller, Quantum Mechanical Transition State Theory and a New Semiclassical Model for Reaction Rate Constants, J. Chem. Phys. 61, 1823 (1974).

[40] W. H. Miller, S. D. Schwartz, and J. W. Tromp, Quantum Mechanical Rate Constants for Bimolecular Reactions, J. Chem. Phys. 79, 4889 (1983).

[41] H. Eyring, The Activated Complex in Chemical Reactions, J. Chem. Phys. 3, 107 (1935).

[42] K. J. Laidler, Chemical Kinetics, 3rd ed. (Harper \& Row, New York, 1987).

[43] R. Chikkaraddy, B. de Nijs, F. Benz, S. J. Barrow, O. A. Scherman, E. Rosta, A. Demetriadou, P. Fox, O. Hess, and J. J. Baumberg, Single-Molecule Strong Coupling at Room Temperature in Plasmonic Nanocavities, Nature (London) 535, 127 (2016).

[44] F. Benz, M. K. Schmidt, A. Dreismann, R. Chikkaraddy, Y. Zhang, A. Demetriadou, C. Carnegie, H. Ohadi, B. de Nijs, R. Esteban, J. Aizpurua, and J. J. Baumberg, SingleMolecule Optomechanics in "Picocavities", Science 354, 726 (2016).

[45] M. Urbieta, M. Barbry, Y. Zhang, P. Koval, D. SánchezPortal, N. Zabala, and J. Aizpurua, Atomic-Scale Lightning Rod Effect in Plasmonic Picocavities: A Classical View to a Quantum Effect, ACS Nano 12, 585 (2018).

[46] M.-K. Kim, H. Sim, S. J. Yoon, S.-H. Gong, C. W. Ahn, Y.-H. Cho, and Y.-H. Lee, Squeezing Photons into a PointLike Space, Nano Lett. 15, 4102 (2015).

[47] S. Scheel and S. Buhmann, Macroscopic Quantum Electrodynamics-Concepts and Applications, Acta Phys. Slovaca 58, 675 (2008).

[48] F. Alpeggiani and L. C. Andreani, Quantum Theory of Surface Plasmon Polaritons: Planar and Spherical Geometries, Plasmonics 9, 965 (2014).

[49] G. Zengin, M. Wersäll, S. Nilsson, T. J. Antosiewicz, M. Käll, and T. Shegai, Realizing Strong Light-Matter Interactions between Single-Nanoparticle Plasmons and Molecular Excitons at Ambient Conditions, Phys. Rev. Lett. 114, 157401 (2015).

[50] R. Chikkaraddy, V. A. Turek, N. Kongsuwan, F. Benz, C. Carnegie, T. van de Goor, B. de Nijs, A. Demetriadou, O. Hess, U. F. Keyser, and J. J. Baumberg, Mapping Nanoscale Hotspots with Single-Molecule Emitters Assembled into Plasmonic Nanocavities Using DNA Origami, Nano Lett. 18, 405 (2018).

[51] M. Autore, P. Li, I. Dolado, F. J. Alfaro-Mozaz, R. Esteban, A. Atxabal, F. Casanova, L. E. Hueso, P. Alonso-González, J. Aizpurua, A. Y. Nikitin, S. Vélez, and R. Hillenbrand, Boron Nitride Nanoresonators for
Phonon-Enhanced Molecular Vibrational Spectroscopy at the Strong Coupling Limit, Light Sci. Appl. 7, 17172 (2018).

[52] C. R. Gubbin, S. A. Maier, and S. De Liberato, Theoretical Investigation of Phonon Polaritons in SiC Micropillar Resonators, Phys. Rev. B 95, 035313 (2017).

[53] T. Niemczyk, F. Deppe, H. Huebl, E. P. Menzel, F. Hocke, M. J. Schwarz, J. J. Garcia-Ripoll, D. Zueco, T. Hümmer, E. Solano, A. Marx, and R. Gross, Circuit Quantum Electrodynamics in the Ultrastrong-Coupling Regime, Nat. Phys. 6, 772 (2010).

[54] P. Forn-Díaz, J. J. García-Ripoll, B. Peropadre, J.-L. Orgiazzi, M. A. Yurtalan, R. Belyansky, C. M. Wilson, and A. Lupascu, Ultrastrong Coupling of a Single Artificial Atom to an Electromagnetic Continuum in the Nonperturbative Regime, Nat. Phys. 13, 39 (2017).

[55] F. Yoshihara, T. Fuse, S. Ashhab, K. Kakuyanagi, S. Saito, and K. Semba, Superconducting Qubit-Oscillator Circuit beyond the Ultrastrong-Coupling Regime, Nat. Phys. 13, 44 (2017).

[56] More precisely, the weak coupling to transversal fields still induces free-space QED effects such as the Lamb shift and radiative decay, but they are not significantly modified by the presence of the cavity.

[57] J. Hopfield, Theory of the Contribution of Excitons to the Complex Dielectric Constant of Crystals, Phys. Rev. 112, 1555 (1958).

[58] B. Huttner and S. M. Barnett, Quantization of the Electromagnetic Field in Dielectrics, Phys. Rev. A 46, 4306 (1992).

[59] H. T. Dung, L. Knöll, and D.-G. Welsch, ThreeDimensional Quantization of the Electromagnetic Field in Dispersive and Absorbing Inhomogeneous Dielectrics, Phys. Rev. A 57, 3931 (1998).

[60] C. Van Vlack, P. T. Kristensen, and S. Hughes, Spontaneous Emission Spectra and Quantum Light-Matter Interactions from a Strongly Coupled Quantum Dot Metal-Nanoparticle System, Phys. Rev. B 85, 075303 (2012).

[61] A. F. Koenderink, On the Use of Purcell Factors for Plasmon Antennas, Opt. Lett. 35, 4208 (2010).

[62] P. T. Kristensen, C. Van Vlack, and S. Hughes, Generalized Effective Mode Volume for Leaky Optical Cavities, Opt. Lett. 37, 1649 (2012).

[63] C. Sauvan, J. P. Hugonin, I. S. Maksymov, and P. Lalanne, Theory of the Spontaneous Optical Emission of Nanosize Photonic and Plasmon Resonators, Phys. Rev. Lett. 110, 237401 (2013).

[64] A. Vukics, T. Grießer, and P. Domokos, Elimination of the A-Square Problem from Cavity QED, Phys. Rev. Lett. 112, 073601 (2014).

[65] D. De Bernardis, P. Pilar, T. Jaako, S. De Liberato, and P. Rabl, Breakdown of Gauge Invariance in UltrastrongCoupling Cavity QED, Phys. Rev. A 98, 053819 (2018).

[66] D. De Bernardis, T. Jaako, and P. Rabl, Cavity Quantum Electrodynamics in the Nonperturbative Regime, Phys. Rev. A 97, 043820 (2018).

[67] V. Rokaj, D. M. Welakuh, M. Ruggenthaler, and A. Rubio, Light-Matter Interaction in the Long-Wavelength Limit: No Ground-State without Dipole Self-Energy, J. Phys. B 51, 034005 (2018). 
[68] A. Stokes and A. Nazir, Gauge Ambiguities Imply JaynesCummings Physics Remains Valid in Ultrastrong Coupling QED, Nat. Commun. 10, 499 (2019).

[69] D. L. Andrews, G. A. Jones, A. Salam, and R. G. Woolley, Perspective: Quantum Hamiltonians for Optical Interactions, J. Chem. Phys. 148, 040901 (2018).

[70] A. Vukics, G. Kónya, and P. Domokos, The GaugeInvariant Lagrangian, the Power-Zienau-Woolley Picture, and the Choices of Field Momenta in Nonrelativistic Quantum Electrodynamics, arXiv:1801.05590v2.

[71] E. Rousseau and D. Felbacq, Reply to "The Equivalence of the Power-Zineau-Woolley Picture and the Poincaré Gauge from the Very First Principles", arXiv:1804.07472.

[72] C. S. Muñoz, F. Nori, and S. De Liberato, Resolution of Superluminal Signalling in Non-Perturbative Cavity Quantum Electrodynamics, Nat. Commun. 9, 1924 (2018).

[73] S. Y. Buhmann, Casimir-Polder Forces on Atoms in the Presence of Magnetoelectric Bodies, $\mathrm{Ph}$. D. thesis, Friedrich-Schiller-Universität Jena, 2007.

[74] T. Neuman, R. Esteban, D. Casanova, F. J. García-Vidal, and J. Aizpurua, Coupling of Molecular Emitters and Plasmonic Cavities beyond the Point-Dipole Approximation, Nano Lett. 18, 2358 (2018).

[75] A. Cuartero-González and A. I. Fernández-Domínguez, Light-Forbidden Transitions in Plasmon-Emitter Interactions beyond the Weak Coupling Regime, ACS Photonics 5, 3415 (2018).

[76] C. Schäfer, M. Ruggenthaler, and A. Rubio, Ab Initio Nonrelativistic Quantum Electrodynamics: Bridging Quantum Chemistry and Quantum Optics from Weak to Strong Coupling, Phys. Rev. A 98, 043801 (2018).

[77] R.-Q. Li, D. Hernángomez-Pérez, F. J. García-Vidal, and A. I. Fernández-Domínguez, Transformation Optics Approach to Plasmon-Exciton Strong Coupling in Nanocavities, Phys. Rev. Lett. 117, 107401 (2016).

[78] K. J. Savage, M. M. Hawkeye, R. Esteban, A. G. Borisov, J. Aizpurua, and J. J. Baumberg, Revealing the Quantum Regime in Tunnelling Plasmonics, Nature (London) 491, 574 (2012).

[79] P. Zhang, J. Feist, A. Rubio, P. García-González, and F. J. García-Vidal, Ab Initio Nanoplasmonics: The Impact of Atomic Structure, Phys. Rev. B 90, 161407(R) (2014).

[80] A. Varas, P. García-González, J. Feist, F. J. García-Vidal, and A. Rubio, Quantum Plasmonics: From Jellium Models to Ab Initio Calculations, Nanophotonics 5, 409 (2016).

[81] S. Shin and H. Metiu, Nonadiabatic Effects on the Charge Transfer Rate Constant: A Numerical Study of a Simple Model System, J. Chem. Phys. 102, 9285 (1995).

[82] S. Shin and H. Metiu, Multiple Time Scale Quantum Wavepacket Propagation: Electron-Nuclear Dynamics, J. Phys. Chem. 100, 7867 (1996).

[83] A. Abedi, F. Agostini, Y. Suzuki, and E. K. U. Gross, Dynamical Steps That Bridge Piecewise Adiabatic Shapes in the Exact Time-Dependent Potential Energy Surface, Phys. Rev. Lett. 110, 263001 (2013).

[84] A. Shalabney, J. George, J. Hutchison, G. Pupillo, C. Genet, and T. W. Ebbesen, Coherent Coupling of Molecular Resonators with a Microcavity Mode, Nat. Commun. 6, 5981 (2015).
[85] B. I. Schneider, J. Feist, S. Nagele, R. Pazourek, S. Hu, L. A. Collins, and J. Burgdörfer, Recent Advances in Computational Methods for the Solution of the Time-Dependent Schrödinger Equation for the Interaction of Short, Intense Radiation with One and Two-Electron Systems: Application to He and $\mathrm{H}_{2}^{+}$, in Quantum Dynamic Imaging, CRM Series in Mathematical Physics, edited by A. D. Bandrauk and M. Ivanov (Springer, New York, 2011), p. 149.

[86] A. González-Tudela, P. A. Huidobro, L. Martín-Moreno, C. Tejedor, and F. J. García-Vidal, Reversible Dynamics of Single Quantum Emitters near Metal-Dielectric Interfaces, Phys. Rev. B 89, 041402(R) (2014).

[87] A. Delga, J. Feist, J. Bravo-Abad, and F. J. Garcia-Vidal, Quantum Emitters near a Metal Nanoparticle: Strong Coupling and Quenching, Phys. Rev. Lett. 112, 253601 (2014).

[88] A. Delga, J. Feist, J. Bravo-Abad, and F. J. Garcia-Vidal, Theory of Strong Coupling between Quantum Emitters and Localized Surface Plasmons, J. Opt. 16, 114018 (2014).

[89] K. D. Bonin and V. V. Kresin, Electric-Dipole Polarizabilities of Atoms, Molecules, and Clusters (World Scientific, Singapore, 1997).

[90] S. D. Fried and S. G. Boxer, Electric Fields and Enzyme Catalysis, Annu. Rev. Biochem. 86, 387 (2017).

[91] V. V. Welborn, L. R. Pestana, and T. Head-Gordon, Computational Optimization of Electric Fields for Better Catalysis Design, Nat. Catal. 1, 649 (2018).

[92] D. M. Bishop, Molecular Vibrational and Rotational Motion in Static and Dynamic Electric Fields, Rev. Mod. Phys. 62, 343 (1990).

[93] D. M. Bishop, Molecular Vibration and Nonlinear Optics, in Advances in Chemical Physics, edited by I. Prigogine and S. A. Rice (Wiley-Blackwell, New York, 1998), p. 1.

[94] R. Cammi, B. Mennucci, and J. Tomasi, Solvent Effects on Linear and Nonlinear Optical Properties of DonorAcceptor Polyenes: Investigation of Electronic and Vibrational Components in Terms of Structure and Charge Distribution Changes, J. Am. Chem. Soc. 120, 8834 (1998).

[95] B. S. Simpkins, K. P. Fears, W. J. Dressick, B. T. Spann, A. D. Dunkelberger, and J. C. Owrutsky, Spanning Strong to Weak Normal Mode Coupling between Vibrational and Fabry-Pérot Cavity Modes through Tuning of Vibrational Absorption Strength, ACS Photonics 2, 1460 (2015).

[96] A. D. Dunkelberger, B. T. Spann, K. P. Fears, B. S. Simpkins, and J.C. Owrutsky, Modified Relaxation Dynamics and Coherent Energy Exchange in Coupled Vibration-Cavity Polaritons, Nat. Commun. 7, 13504 (2016).

[97] H. B. G. Casimir and D. Polder, The Influence of Retardation on the London-van Der Waals Forces, Phys. Rev. 73, 360 (1948).

[98] A. Stone, The Theory of Intermolecular Forces, 2nd ed. (Oxford University, New York, 2013).

[99] A. Lombardi, M. K. Schmidt, L. Weller, W. M. Deacon, F. Benz, B. de Nijs, J. Aizpurua, and J. J. Baumberg, Pulsed Molecular Optomechanics in Plasmonic Nanocavities: From Nonlinear Vibrational Instabilities to Bond-Breaking, Phys. Rev. X 8, 011016 (2018). 
[100] A. D. Becke, Density-Functional Thermochemistry. III. The Role of Exact Exchange, J. Chem. Phys. 98, 5648 (1993).

[101] C. M. Isborn, N. Luehr, I. S. Ufimtsev, and T. J. Martínez, Excited-State Electronic Structure with Configuration Interaction Singles and Tamm-Dancoff Time-Dependent Density Functional Theory on Graphical Processing Units, J. Chem. Theory Comput. 7, 1814 (2011).

[102] I. S. Ufimtsev and T. J. Martinez, Quantum Chemistry on Graphical Processing Units. 3. Analytical Energy Gradients, Geometry Optimization, and First Principles Molecular Dynamics, J. Chem. Theory Comput. 5, 2619 (2009).

[103] A. V. Titov, I. S. Ufimtsev, N. Luehr, and T. J. Martinez, Generating Efficient Quantum Chemistry Codes for Novel Architectures, J. Chem. Theory Comput. 9, 213 (2013).

[104] C. I. Sukenik, M. G. Boshier, D. Cho, V. Sandoghdar, and E. A. Hinds, Measurement of the Casimir-Polder Force, Phys. Rev. Lett. 70, 560 (1993).

[105] J. George, A. Shalabney, J. A. Hutchison, C. Genet, and T. W. Ebbesen, Liquid-Phase Vibrational Strong Coupling, J. Phys. Chem. Lett. 6, 1027 (2015).

[106] B. M. Axilrod and E. Teller, Interaction of the van der Waals Type between Three Atoms, J. Chem. Phys. 11, 299 (1943).

[107] C. Nicosia and J. Huskens, Reactive Self-Assembled Monolayers: From Surface Functionalization to Gradient Formation, Mater. Horiz. 1, 32 (2014).

[108] G. P. Acuna, F. M. Möller, P. Holzmeister, S. Beater, B. Lalkens, and P. Tinnefeld, Fluorescence Enhancement at Docking Sites of DNA-Directed Self-Assembled Nanoantennas, Science 338, 506 (2012).

[109] S. Kéna-Cohen, M. Davanço, and S. R. Forrest, Strong Exciton-Photon Coupling in an Organic Single Crystal Microcavity, Phys. Rev. Lett. 101, 116401 (2008).

[110] J. Hulliger, T. Wüst, K. Brahimi, and J. C. Martinez Garcia, Can Mono Domain Polar Molecular Crystals Exist?, Cryst. Growth Des. 12, 5211 (2012).

[111] M. Hertzog, P. Rudquist, J. A. Hutchison, J. George, T. W. Ebbesen, and K. Börjesson, Voltage-Controlled Switching of Strong Light-Matter Interactions Using Liquid Crystals, Chem.-Eur. J. 23, 18166 (2017).

[112] We additionally checked explicitly that solving the full electrostatic problem, i.e., including all modes of the sphere by using the method of image dipoles, gives very similar results to the ones presented here.
[113] Free Energy Calculations, Springer Series in Chemical Physics Vol. 86, edited by C. Chipot, A. Pohorille, A. W. Castleman, J. P. Toennies, K. Yamanouchi, and W. Zinth (Springer, Berlin, 2007).

[114] J. I. Mendieta-Moreno, D. G. Trabada, J. Mendieta, J. P. Lewis, P. Gómez-Puertas, and J. Ortega, Quantum Mechanics/Molecular Mechanics Free Energy Maps and Nonadiabatic Simulations for a Photochemical Reaction in DNA: Cyclobutane Thymine Dimer, J. Phys. Chem. Lett. 7, 4391 (2016).

[115] E. Cortese, P. G. Lagoudakis, and S. De Liberato, Collective Optomechanical Effects in Cavity Quantum Electrodynamics, Phys. Rev. Lett. 119, 043604 (2017).

[116] S. Y. Buhmann and S. Scheel, Thermal Casimir versus Casimir-Polder Forces: Equilibrium and Nonequilibrium Forces, Phys. Rev. Lett. 100, 253201 (2008).

[117] S. Å. Ellingsen, S. Y. Buhmann, and S. Scheel, Temperature-Independent Casimir-Polder Forces Despite Large Thermal Photon Numbers, Phys. Rev. Lett. 104, 223003 (2010).

[118] K. Sinha, B. P. Venkatesh, and P. Meystre, Collective Effects in Casimir-Polder Forces, Phys. Rev. Lett. 121, 183605 (2018).

[119] C. Climent, J. Galego, F. J. Garcia-Vidal, J. Feist, Plasmonic Nanocavities Enable Self-Induced Electrostatic Catalysis, Angew. Chem. Int. Ed., http://dx.doi.org/10 .1002/anie.201901926 (2019).

[120] E. Waks and D. Sridharan, Cavity QED Treatment of Interactions between a Metal Nanoparticle and a Dipole Emitter, Phys. Rev. A 82, 043845 (2010).

[121] C. Gonzalez-Ballestero, J. Feist, E. Moreno, and F. J. Garcia-Vidal, Harvesting Excitons through Plasmonic Strong Coupling, Phys. Rev. B 92, 121402(R) (2015).

[122] P. Anger, P. Bharadwaj, and L. Novotny, Enhancement and Quenching of Single-Molecule Fluorescence, Phys. Rev. Lett. 96, 113002 (2006).

[123] P. de Vries, D. van Coevorden, and A. Lagendijk, Point Scatterers for Classical Waves, Rev. Mod. Phys. 70, 447 (1998).

[124] J. del Pino, J. Feist, and F. J. Garcia-Vidal, Signatures of Vibrational Strong Coupling in Raman Scattering, J. Phys. Chem. C 119, 29132 (2015).

[125] D. P. Craig and T. Thirunamachandran, Molecular Quantum Electrodynamics: An Introduction to RadiationMolecule Interactions (Courier, North Chelmsford, MA, 1998). 